\title{
Minimum divergence viscous flow simulation through finite difference and regularization techniques
}

\author{
Rodolfo A Victor ${ }^{\mathrm{a}, \mathrm{b}, *}$, Maryam Mirabolghasemi ${ }^{\mathrm{b}}$, Steven L Bryant ${ }^{\mathrm{c}}$, Maša Prodanović ${ }^{\mathrm{b}}$ \\ ${ }^{a}$ Petróleo Brasileiro S.A., Petrobras, Rio de Janeiro, RJ, Brazil \\ ${ }^{b}$ Department of Petroleum and Geosystems Engineering, University of Texas at Austin, Austin, TX, \\ $U S A$ \\ ${ }^{c}$ Schulich School of Engineering, University of Calgary, Calgary, Alberta, Canada
}

\begin{abstract}
We develop a new algorithm to simulate single- and two-phase viscous flow through a three-dimensional Cartesian representation of the porous space, such as those available through X-ray microtomography. We use the finite difference method to discretize the governing equations and also propose a new method to enforce the incompressible flow constraint under zero Neumann boundary conditions for the velocity components. Finite difference formulation leads to fast parallel implementation through linear solvers for sparse matrices, allowing relatively fast simulations, while regularization techniques used on solving inverse problems lead to the desired incompressible fluid flow.

Tests performed using benchmark samples show good agreement with experimental/theoretical values. Additional tests are run on Bentheimer and Buff Berea sandstone samples with available laboratory measurements. We compare the results from our new method, based on finite differences, with an open source finite volume implementation as well as experimental results, specifically to evaluate the benefits and drawbacks of each method. Finally, we calculate relative permeability by using this modified finite difference technique together with a level set based algorithm for multi-phase fluid distribution in the pore space. To our knowledge this is the first time regularization techniques are used in combination with finite difference fluid flow simulations.
\end{abstract}

Keywords:

Fluid flow in porous media, finite difference method, finite volume method, absolute permeability, relative permeability, Tikhonov regularization

\section{Introduction}

Determination and prediction of porous rocks' macroscopic properties form an essential part of the workflow in oil and gas industry, from exploration geophysics and

\footnotetext{
* Corresponding author

Email addresses: rodolfoavictor@utexas.edu (Rodolfo A Victor), Maryam.m@utexas.edu (Maryam Mirabolghasemi), Steven.Bryant@ucalgary.ca (Steven L Bryant), masa@utexas.edu (Maša Prodanović)

Preprint submitted to Advances in Water Resources

February 1, 2016
} 
formation evaluation to reservoir and production engineering. Due to the lack of rigorous relations between the rock pore space and the corresponding petrophysical behavior, methods for predicting rock's responses to gradients in potential have strongly relied on empirical correlations, whose fitting parameters are adjusted for a particular rock type using laboratory measurements. Two famous representatives of these correlations are the Archie's equation [1], relating the rock electrical conductivity to water saturation, and the widely used power laws relating porosity and permeability (see, for example, Peters [2], Chapter 3). The use of empirical methods, although somewhat successful when targeting the right set of rocks, often do not provide any insight into the fundamental physical principles relating the pore geometry and the macroscopic behavior of the rock.

Over the past decades, many approximate methods were proposed by representing rocks using idealized models such as bundle of capillary tubes or disordered pack of spheres geometrically modified to simulate geologic processes [3-5]. Although these systems are much simpler than the actual porous media, the resulting equations showed a good performance on predicting physical properties of simpler rocks like clean sandstones [6]. However, general relations for rocks like carbonates and unconventional hydrocarbon reservoirs are a formidable task still to be completed.

A fast growing research field for investigating processes at pore-level scale uses the digital rock physics approach $[7,8]$. Using modern experimental methods to acquire high resolution 3D images of rock samples, a detailed model of the complex pore geometry is constructed and several physical processes with different scenarios can then be simulated and studied. This approach also has the advantage of providing valuable insight towards a fundamental understanding of the governing pore-scale processes.

The workflow in digital petrophysics is formed by three main steps. First, a 3D porescale image of the rock sample is acquired. The most used imaging technique is the X-ray computed micro-tomography ( $\mu$-CT), which measures the local X-ray absorption inside the sample and translates this information to a $3 \mathrm{D}$ gray scale image. The technique was introduced by Flannery et al. [9] and recent reviews are provided by Wildenschild and Sheppard [8], and Cnudde and Boone [10].

The second and probably most critical step is to classify each voxel as a mineral phase, a fluid phase or a combination of both, depending on the image resolution. This step, called segmentation, is usually preceded by some pre-processing for quality enhancement. Comprehensive reviews on the available segmentation methods are provided by the works of Iassonov et al. [11] and Schlüter et al. [12].

The final step is the pore space characterization and simulation of the physical processes using the segmented image. This step leads to an estimate of the rock's bulk behavior and its petrophysical properties, but also gives the opportunity to test different system configurations and validate theoretical models through comparison between simulation results and laboratory measurements. There are published works simulating several different properties, for example electrical conductivity and formation factor [13-18], elastic moduli [17-21] and nuclear magnetic resonance response [5, 22-25].

One of the first approaches in simulating single-phase velocity and hydraulic absolute permeability uses network modeling. This technique represents the pore space as a network of sites, corresponding to pore bodies, connected by bonds, corresponding to pore throats. Macroscopic properties can then be estimated by applying the pertinent conservation equations describing the phenomena. Pore networks using data from Finney [3], who experimentally measured the spatial position of approximately 8000 precision 
ball bearings in a dense disordered pack, showed good performance in predicting trends in transport properties for simple sandstones that have undergone the same geologic processes [6, 26, 27]. Modern modeling now starts from 3D images of rock samples, and a review on related methods is provided by Blunt et al. [28, 29]. Recent works have applied pore network modeling to investigate unconventional resources like tightgas sandstones [30,31] and shale gas [32]. Network modeling, although computationally cheaper, simplifies the velocity field within the pore space. An intermediate solution is given by Shabro et al. [33], where the pore voxels are divided into interconnected cylindrical pores, each one with a flow described by the Hagen-Poiseuille equation. The overall flow rate is then achieved by weighting the mass flux in each cylinder using the distance from the cylinder to the nearest grain voxel.

In the last decade, increasing computational resources have made possible the application of direct simulation methods in 3D images. The first established method to simulate fluid flow at pore scale in complex geometries is the lattice-Boltzmann method $[34,35]$. In this approach, the fluid is modeled by a collection of particles following simple rules for local interactions, recovering the Navier-Stokes equation for the macroscopic fluid behavior. The absolute permeability is then estimated using Darcy's equation after calculating the inlet and the outlet pressures. This method provides a rigorous estimate of flow properties, since there is no additional simplification to the pore space. Results for estimating absolute permeability using lattice-Boltzmann are widely available in the literature $[15,17,18,36]$.

Another modeling approach is to solve the integral form of the conservation laws. This approach is the basis of finite element (FEM) and finite volume (FVM) methods $[37,38]$. Different formulations of this class have been proposed to efficiently solve flow equations in geometries consisting of hundreds of pores [39, 40], usually extracted from $\mu$-CT images of porous samples, and they have been proved useful in solving full NavierStokes equations in porous media. Icardi et al. [41] employed a finite volume scheme to simulate the flow field in virtual polydisperese packing of irregular objects. While they mainly focused on spatial discretization issues, they also examined the Eulerian approach to model solute transport in porous media. A common challenge associated with FEM/FVM is the quality of domain discretization. Further, precise modeling of the momentum transfer between the fluid and solid grains is the key to estimate the correct permeability of the porous media. However, the complex structure of solid surface in a porous sample makes it very difficult to model all fluid-solid interactions in detail.

Despite the above-mentioned challenges, FEM/FVM have been successfully used to characterize the hydraulic properties of rocks. Akanji and Matthai [39] developed a fast two-step FEM solution to calculate the permeability of different rock samples. They computed the flow field in samples of Ottawa sandstone, LV60 sandstone, and Sombrero Beach carbonate sand and validated the resulting permeability values against experimental values. FEM has also been proved useful in modeling viscoelastic flows. Viscoelasticity is known to introduce oscillation in numerical solutions of the flow field. Falco et al. [40] used a stabilized FEM to simulate viscoelastic polymer flow in replicated sandstone scanning electron microscopy images and validated their model using previously published data.

The finite difference method (FDM) also forms a well established discretization technique for computational fluid dynamics. It has been used to estimate permeability in digitized porous media [42, 43], including multiphase flow simulations [44]. It allows 
very efficient use of computational resources, leading to the possibility of investigating larger and more representative samples. The standard workflow directly solves Stokes or Navier-Stokes equation, generating a detailed velocity field for the pore space. Permeability is then calculated via Darcy's equation and velocity field volumetric averages. Staggered grid, with pressure defined at the voxel center and velocity components defined at the voxel faces, has been the common approach, from early works like Schwartz et al. [45], relating electrical conductivity and hydraulic permeability in packings of spherical grains, until more recent fluid flow simulations using $\mu$-CT images from real rocks $[13,42-44]$. The use of FDM with collocated (non-staggered) grid has also been reported $[46,47]$.

The problem of choosing appropriate boundary conditions for FDM implementation of incompressible Stokes flow has been revisited a number of times [48-52]. The existence and uniqueness of the problem of incompressible flow under Dirichlet pressure boundary conditions requires adding restrictions on velocities at the inlet and outlet boundaries [53]. Different methods have been proposed to tackle this limitation: high order finite difference schemes [54]; an iterative pressure correction algorithm with periodic velocity boundary conditions [43]; artificial compressibility with zero spatial derivative velocity boundary conditions $[44,55]$; and treating boundary nodes as internal nodes for normal velocity with zero tangent velocity and the explicit method of incomplete approximation [53]. Immerse boundary method [56-58] was introduced in biological fluid mechanics to handle the interaction of incompressible fluid and elastic boundaries, and has been used to simulate fluid flow in porous media $[59,60]$.

In this paper we propose the use of FDM together with regularization techniques from the theory of inverse problems to pursue a small divergence solution for the velocity field in porous media. We validate our results for velocity and absolute permeability using benchmark samples, whose properties are either theoretically known or measured in laboratory. We also compare the results of absolute permeability with finite volume simulations. To our knowledge this is the first time regularization techniques are used in combination with finite difference fluid flow simulation techniques.

Accurate multi-phase fluid distribution on the pore space is an important step in the simulation of multi-phase fluid properties. Some methods that handle fluid distribution in pore space are available in the literature. Most assume capillarity controls the configuration of the flowing phases, an idea that dates back to Fattès' introduction of the network model. For example, the method of maximal inscribed spheres [61] assigns to each voxel the radius of the maximal sphere included in the pore space and covering that voxel. Invasion scenarios are then created by cluster search on these radius map. This method is used by Silin and Patzek to calculate relative permeability [44]. Another similar method is called the capillary drainage transform [62], which simulates drainage by letting a non-wetting phase invade a pore region if a sphere with radius given by Young-Laplace equation can be moved from a non-wetting saturated region to the target region. Capillary drainage transform is used to calculate resistivity index in [14, 63, 64].

Advances in computational resources have allowed the modeling of multi-phase flow and even hybrid multi-scale problems through the finite volume formulation. Tomin and Lunati [65] developed a hybrid multiscale finite volume method that enables coupling of pore-scale and Darcy-scale multi-phase flow in porous media. Their model benefits from a local pressure gradient and flux relationship, which is calculated from solving Navier-Stokes equations on the pore-scale. At the coarse-scale, a volume of fluid model 
was used to track the interface between the two phases. Two-phase relative permeability estimated by lattice-Boltzmann has also been reported [66], but to date this approach is still computationally expensive.

Prodanović and Bryant [67] introduced the level set based progressive quasi-static algorithm (LSMPQS), to find capillary-dominated two-phase fluid distribution in 3D images of pore space. Level sets formulation is used to move the fluid-fluid interface through the porous medium, with Young-Laplace equation enforced at each equilibrium position. The distinctive feature of this approach is that it is general for both drainage and imbibition. LSMPQS successfully matches experimentally observed trapped nonwetting phase, although it does not provide the flow field [68-70]. In this paper we exemplify the use of our FDM implementation together with LSMPQS to investigate relative permeability from the results of drainage processes.

\section{Materials and methods}

\subsection{Governing equations for single-phase flow}

The velocity field for a single-phase fluid flow is governed by two equations of change [71]. First, the equation of continuity,

$$
\frac{\partial \rho}{\partial t}+\vec{\nabla} \cdot \rho \vec{v}=0,
$$

where $\rho(x, y, z, t)$ is the fluid density and $\vec{v}(x, y, z, t)$ is the fluid velocity. Our simulations will consider incompressible flow ( $\rho$ constant), which reduces the equation of continuity to

$$
\vec{\nabla} \cdot \vec{v}=0 .
$$

Second, the equation of motion, which for a Newtonian fluid with viscosity $\mu$ and density $\rho$ reduces to the Navier-Stokes equation

$$
\rho \frac{D}{D t} \vec{v}=-\vec{\nabla} p+\mu \nabla^{2} \vec{v}+\rho \vec{g} .
$$

Here $D / D t=\partial / \partial t+\vec{v} \cdot \vec{\nabla}$ is the material derivative, $p(x, y, z, t)$ is the fluid pressure and $\vec{g}$ is the gravitational acceleration. Pressure and gravity terms in the above equation can be conveniently lumped together as the so-called modified pressure

$$
\mathscr{P}=p+\rho g h,
$$

where $h$ is the distance measured in a direction opposite to gravity from some reference point. In this notation, Navier-Stokes equation is written as

$$
\rho \frac{D}{D t} \vec{v}=-\vec{\nabla} \mathscr{P}+\mu \nabla^{2} \vec{v} .
$$

We will simply refer to $\mathscr{P}$ as pressure.

Solving Navier-Stokes equations is much harder when the advective term $\rho D \vec{v} / D t$ is dominant. However, due to the small length scales involved in flow through a porous medium, incompressible flow regimes are usually in the low Reynolds number laminar 
steady state. In other words, the assumption of creeping flow holds true for most flows in porous media. This enables describing the steady state flow by the Stokes equation,

$$
\nabla^{2} \vec{v}=\frac{1}{\mu} \vec{\nabla} \mathscr{P},
$$

which is linear and much easier to solve numerically.

The pressure field can be decoupled from Equation (6) by taking its divergence,

$$
\vec{\nabla} \cdot\left(\mu \nabla^{2} \vec{v}\right)=\nabla^{2} \mathscr{P},
$$

and applying the vector identity

$$
\vec{\nabla} \times \vec{\nabla} \times \vec{v}=\vec{\nabla}(\vec{\nabla} \cdot \vec{v})-\nabla^{2} \vec{v}
$$

to obtain

$$
\mu \vec{\nabla} \cdot(\vec{\nabla}(\vec{\nabla} \cdot \vec{v})-\vec{\nabla} \times \vec{\nabla} \times \vec{v})=\nabla^{2} \mathscr{P} .
$$

Since $\vec{\nabla} \cdot \vec{v}=0$ from Equation (2) and the divergence of a rotational is identically zero, we get a Laplace equation for the pressure field in the laminar regime:

$$
\nabla^{2} \mathscr{P}=0 .
$$

Equations (2), (6), and (10) can be solved for the porous medium by defining an appropriate set of boundary conditions. Given a Cartesian $x y z$ coordinate system, we assume the flow can enter and exit the porous medium only through the first and last $x y$ planes, driving the macroscopic flow in the $z$ direction.

As shown by Whitaker [72], the volumetric flow rate

$$
q=\oint \vec{v} \cdot \hat{n} d A
$$

of an incompressible single-phase fluid in laminar flow through a porous medium averages to Darcy's law

$$
q=\frac{\kappa A}{\mu} \frac{\Delta \mathscr{P}}{L},
$$

where $A$ is the area of a cross-section perpendicular to the flow direction, $L$ is the length of the sample and $\kappa$ is the absolute permeability of the medium. Thus, our simulation results can be compared with real measurements by combining those two equations in

$$
\kappa_{z}=\frac{\mu L}{A \Delta \mathscr{P}} \oint v_{z} d A .
$$

\subsection{Finite difference implementation}

We chose the 7-point stencil finite difference method [73] to discretize the equations governing the transport of fluid on a three-dimensional Cartesian grid, with both pressure and velocity calculated at the center of the voxel. We will represent the number of voxels in each direction by $n_{x}, n_{y}$, and $n_{z}$, with respective voxel lengths $h_{x}, h_{y}$, and $h_{z}$. 
FDM has several advantages over other methods. First, it uses the segmented image without any further processing, as opposed to the FEM/FVM method, where a representative mesh usually has to be generated to feed the simulator.

Second, detailed representation for all physical quantities in the pore space is a natural consequence when using voxel-based computational grid. In pore-network modeling, for example, generating a network reflecting the specific properties of an arbitrary porous medium is still a challenge $[6,28,32,74]$.

Further, boundary conditions such as no-slip at pore-grain contact and fixed pressure at the open boundaries are straightforward to implement. Our approach, to be detailed below, treats the boundaries directly on the voxelized grids, which is preferred for applicability in imaged porous media. This eliminates the need of additional analysis at pore-grain boundaries. A rigorous implementation of these conditions may not be as simple in lattice-Boltzmann simulations.

Finally, a careful implementation of finite differences can lead to computationally fast and memory efficient algorithms. It can be a promising alternative, for example, for lattice-Boltzman simulations of two-phase flow with the interfacial phenomena, which so far still require massive computations.

The main disadvantage of finite differences with open flow boundary conditions is the residual divergence at the velocity field, which can be significant in some geometries. We address this problem later in this section.

\subsubsection{Linear equations for pressure field}

Let $i, j$, and $k$ be the corresponding voxel coordinates in $x, y$, and $z$ directions, such that $1 \leq i \leq n_{x}, 1 \leq j \leq n_{y}$ and $1 \leq k \leq n_{z}$. For the sake of simplicity in the notation, let us also introduce $n_{x y}=n_{x} n_{y}$ and $n_{x y z}=n_{x} n_{y} n_{z}$.

The second order central finite difference approximation of Equation (10) for the voxel at position $(i, j, k)$ is given by

$$
\begin{aligned}
\frac{\mathscr{P}_{i+1, j, k}-2 \mathscr{P}_{i, j, k}+\mathscr{P}_{i-1, j, k}}{h_{x}^{2}}+\frac{\mathscr{P}_{i, j+1, k}-2 \mathscr{P}_{i, j, k}+\mathscr{P}_{i, j-1, k}}{h_{y}^{2}} & \\
+\frac{\mathscr{P}_{i, j, k+1}-2 \mathscr{P}_{i, j, k}+\mathscr{P}_{i, j, k-1}}{h_{z}^{2}} & =0 .
\end{aligned}
$$

The set of the above equations for each voxel in the $3 \mathrm{D}$ grid forms a system of $n_{x y z}$ equations and $n_{x y z}$ variables. In order to use the formalism of matrix algebra, we introduce the index

$$
K=(k-1) n_{x y}+(j-1) n_{x}+i .
$$

This puts the $n_{x y z}$ equations in the form

$$
\begin{aligned}
\frac{1}{h_{z}^{2}} \mathscr{P}_{K+n_{x y}}+\frac{1}{h_{y}^{2}} \mathscr{P}_{K+n_{x}}+\frac{1}{h_{x}^{2}} \mathscr{P}_{K+1}-\left(\frac{2}{h_{x}^{2}}+\frac{2}{h_{y}^{2}}+\frac{2}{h_{z}^{2}}\right) \mathscr{P}_{K} \\
+\frac{1}{h_{x}^{2}} \mathscr{P}_{K-1}+\frac{1}{h_{y}^{2}} \mathscr{P}_{K-n_{x}}+\frac{1}{h_{z}^{2}} \mathscr{P}_{K-n_{x y}}=0 .
\end{aligned}
$$




\subsubsection{Boundary conditions for pressure field}

Dirichlet boundary conditions are implemented at the $z$ borders by modifying the corresponding equations to set a constant pressure value at those voxels. If $k=1$, Equation (16) reduces to

$$
\mathscr{P}_{K}=\mathscr{P}_{\text {in }},
$$

while if $k=n_{z}$ we get

$$
\mathscr{P}_{K}=\mathscr{P}_{\text {out }},
$$

for some user-defined $\mathscr{P}_{\text {in }}$ and $\mathscr{P}_{\text {out }}$. Voxels with $i=1, j=1, i=n_{x}$, and $j=n_{y}$ are set to grain phase to enforce closed boundaries in $x$ and $y$ directions.

The governing equations resulting from the assumption of fluid incompressibility and low Reynolds number regime lead to a heuristic method to set the boundary conditions for pressure at pore-grain contacts. Let $V$ be the volume of a pore region enclosed by a surface of area $A$. Equation (10) can be written as

$$
\vec{\nabla} \cdot \vec{\nabla} \mathscr{P}=0,
$$

which, after applying the divergence theorem, becomes

$$
\int_{V}(\vec{\nabla} \cdot \vec{\nabla} \mathscr{P}) d V=\oint_{A}(\vec{\nabla} \mathscr{P} \cdot \hat{n}) d A=0 .
$$

We then impose

$$
\vec{\nabla} \mathscr{P} \cdot \hat{n}=\frac{\partial \mathscr{P}}{\partial n}=0
$$

at the closed boundaries, which is widely used in numerical fluid flow simulations [33, 75]. If we now consider $V$ as the whole sample with closed borders in $x$ and $y$ directions, we can write

$$
\begin{aligned}
\oint_{A}(\vec{\nabla} \mathscr{P} \cdot \hat{n}) d A=\iint_{x=0}\left(-\frac{\partial \mathscr{P}}{\partial x}\right) d y d z+\iint_{x=x_{\max }} \frac{\partial \mathscr{P}}{\partial x} d y d z \\
+\iint_{y=0}\left(-\frac{\partial \mathscr{P}}{\partial y}\right) d x d z+\iint_{y=y_{\max }} \frac{\partial \mathscr{P}}{\partial y} d x d z \\
\quad+\iint_{z=0}\left(-\frac{\partial \mathscr{P}}{\partial z}\right) d x d y+\iint_{z=z_{\max }} \frac{\partial \mathscr{P}}{\partial z} d x d y=0,
\end{aligned}
$$

which implies

$$
\iint_{z=0} \frac{\partial \mathscr{P}}{\partial z} d x d y=\iint_{z=z_{\max }} \frac{\partial \mathscr{P}}{\partial z} d x d y
$$

given the close boundaries in $x$ and $y$ directions. In fact, repeating this analysis for an arbitrary subset of $x y$ slices, we conclude that the flux of the pressure gradient through the sample in $z$ direction,

$$
\Phi_{\vec{\nabla} \mathscr{P}}\left(z_{k}\right)=\iint_{z=z_{k}}(\vec{\nabla} \mathscr{P} \cdot \hat{z}) d x d y=\iint_{z=z_{k}} \frac{\partial \mathscr{P}}{\partial z} d x d y,
$$


is constant through the porous medium. We will later use this property to set a quality control for the simulation results.

The implementation of zero normal derivative is straightforward using the central difference technique. For example, if the normal direction at pore-grain interface is parallel to $\hat{x}+\hat{y}$, we have

$$
\frac{\partial \mathscr{P}}{\partial n}=\vec{\nabla} \mathscr{P} \cdot \hat{n}=\vec{\nabla} \mathscr{P} \cdot\left(\frac{\hat{x}+\hat{y}}{\sqrt{2}}\right)=\frac{1}{\sqrt{2}}\left(\frac{\partial \mathscr{P}}{\partial x}+\frac{\partial \mathscr{P}}{\partial y}\right)=0
$$

and, by finite differences,

$$
\begin{gathered}
\frac{\partial \mathscr{P}}{\partial x}+\frac{\partial \mathscr{P}}{\partial y}=0, \\
\frac{\mathscr{P}_{K+1}-\mathscr{P}_{K-1}}{2 h_{x}}+\frac{\mathscr{P}_{K+n_{x}}-\mathscr{P}_{K-n_{x}}}{2 h_{y}}=0,
\end{gathered}
$$

which is enforced by setting $\mathscr{P}_{K+1}=\mathscr{P}_{K-1}$ and $\mathscr{P}_{K+n_{x}}=\mathscr{P}_{K-n_{x}}$. Since positions $K-1$ and $K-n_{x}$ correspond to grain voxels in this case, Equation (16) is rewritten as

$$
\begin{aligned}
& \frac{1}{h_{z}^{2}} \mathscr{P}_{K+n_{x y}}+\frac{2}{h_{y}^{2}} \mathscr{P}_{K+n_{x}}+\frac{2}{h_{x}^{2}} \mathscr{P}_{K+1} \\
&-\left(\frac{2}{h_{x}^{2}}+\frac{2}{h_{y}^{2}}+\frac{2}{h_{z}^{2}}\right) \mathscr{P}_{K}+\frac{1}{h_{z}^{2}} \mathscr{P}_{K-n_{x y}}=0 .
\end{aligned}
$$

In a second example, if a pore voxel at grid position $(i, j, k)$ has neighbor grain voxels at positions $(i-1, j, k),(i, j+1, k)$ and $(i, j, k-1)$, the corresponding equation for pressure is obtained by eliminating the terms arising from those grain voxels and counting twice the terms arising from the opposing pore voxels, in such a way that Equation (16) becomes, in this case,

$$
\frac{2}{h_{z}^{2}} \mathscr{P}_{K+n_{x y}}+\frac{2}{h_{x}^{2}} \mathscr{P}_{K+1}-\left(\frac{2}{h_{x}^{2}}+\frac{2}{h_{y}^{2}}+\frac{2}{h_{z}^{2}}\right) \mathscr{P}_{K}+\frac{2}{h_{y}^{2}} \mathscr{P}_{K-n_{x}}=0 .
$$

Similar modifications happen for pore-grain contacts aligned in different directions.

\subsubsection{Implementation of pressure field solver}

The system of $n_{x y z}$ linear equations for pressure can now be described by the traditional matrix form $\mathbf{A x}=\mathbf{b}$, where $\mathbf{A}$ is the coefficient matrix for the Laplacian operator, $\mathbf{x}=\left[\mathscr{P}_{1}, \mathscr{P}_{2}, \ldots, \mathscr{P}_{n_{x y z}}\right]^{T}$ is the vector of unknown pressures and $\mathbf{b}$ handles the Dirichlet boundary conditions. The heptadiagonal sparse nature of the coefficient matrix $\mathbf{A}$ makes this system of equations suitable for iterative solution methods [76] without the need of storing matrix A, allowing to efficiently handle bigger samples. Our implementation uses the biconjugate gradient stabilized method with Jacobi preconditioner, whose details can be found in the work of Van der Vorst [77]. Given the boundary conditions, a constant gradient is used as the initial guess for the pressure field solution.

After convergence is achieved, we use Equation (24) as an independent quality control: one standard deviation of $\Phi_{\vec{\nabla} \mathscr{P}}(z)$ divided by its mean value, at the pore voxels, is defined as a measure of relative numerical uncertainty for the pressure field. 


\subsubsection{Linear equations for velocity field}

Once the pressure field is calculated, it is used as a forcing function to solve Poisson's equation for velocity. In principle, Equation (6) can be solved separately in each direction, leading to a set of Poisson equations for the scalar velocity components $v_{x}, v_{y}$ and $v_{z}$,

$$
\begin{aligned}
\nabla^{2} v_{x} & =\frac{1}{\mu} \frac{\partial \mathscr{P}}{\partial x}, \\
\nabla^{2} v_{y} & =\frac{1}{\mu} \frac{\partial \mathscr{P}}{\partial y}, \\
\nabla^{2} v_{z} & =\frac{1}{\mu} \frac{\partial \mathscr{P}}{\partial z},
\end{aligned}
$$

whose discretization would be analogous to the pressure case, Equation (16). Pressure gradients are calculated by central finite difference scheme,

$$
\begin{aligned}
\frac{\partial \mathscr{P}}{\partial x} & \approx \frac{\mathscr{P}_{i+1, j, k}-\mathscr{P}_{i-1, j, k}}{2 h_{x}}, \\
\frac{\partial \mathscr{P}}{\partial y} & \approx \frac{\mathscr{P}_{i, j+1, k}-\mathscr{P}_{i, j-1, k}}{2 h_{y}}, \\
\frac{\partial \mathscr{P}}{\partial z} & \approx \frac{\mathscr{P}_{i, j, k+1}-\mathscr{P}_{i, j, k-1}}{2 h_{z}} .
\end{aligned}
$$

\subsubsection{Boundary conditions for velocity field}

No slip and no fluid penetration boundary conditions are imposed by simply setting zero velocity at the grain voxels. For boundary $z$ voxels, one common approach is to specify a velocity profile at either the inlet or the outlet open boundary, but this can lead to artificial flow configurations in complex pore spaces. Since we already set Dirichlet conditions for pressure, we use zero Neumann boundary conditions for velocity, setting its directional derivatives to zero at the open boundaries. However, even if the pressure field is estimated using the zero divergence condition for velocity, its use as a forcing function does not guarantee a zero divergence velocity field, as also pointed by Nördstrom et al. [52]. Moreover, although the fully developed flow profile can be achieved by this set of boundary conditions, they also lead to a system with non-unique solution.

\subsubsection{Minimizing the velocity field divergence}

We use a different approach to couple Stokes equation and the zero divergence constraint based on techniques from the theory of inverse problems. Let $\mathbf{A}$ be the Laplacian operator for the velocity components, $\mathbf{b}_{\mathbf{x}}, \mathbf{b}_{\mathbf{y}}$, and $\mathbf{b}_{\mathbf{z}}$ be respectively the pressure gradient in $x, y$, and $z$ directions, divided by viscosity and indexed as in Equation (15), and D be the divergence operator matrix formed from the set of equations

$$
\vec{\nabla} \cdot \vec{v}(x, y, z) \approx \frac{v_{x}^{K+1}-v_{x}^{K-1}}{2 h_{x}}+\frac{v_{y}^{K+n_{x}}-v_{y}^{K-n_{x}}}{2 h_{y}}+\frac{v_{z}^{K+n_{x y}}-v_{z}^{K-n_{x y}}}{2 h_{y}} .
$$

The problem can now be reformulated as to solve the $3 n_{x y z} \times 3 n_{x y z}$ system formed by the block matrices

$$
\left[\begin{array}{ccc}
\mathbf{A}_{x} & \mathbf{0} & \mathbf{0} \\
\mathbf{0} & \mathbf{A}_{y} & \mathbf{0} \\
\mathbf{0} & \mathbf{0} & \mathbf{A}_{z}
\end{array}\right]\left[\begin{array}{c}
\mathbf{v}_{\mathbf{x}} \\
\mathbf{v}_{\mathbf{y}} \\
\mathbf{v}_{\mathbf{z}}
\end{array}\right]=\left[\begin{array}{c}
\mathbf{b}_{\mathbf{x}} \\
\mathbf{b}_{\mathbf{y}} \\
\mathbf{b}_{\mathbf{z}}
\end{array}\right]
$$


subject to

$$
\mathbf{D}\left[\begin{array}{c}
\mathbf{v}_{\mathbf{x}} \\
\mathbf{v}_{\mathbf{y}} \\
\mathbf{v}_{\mathbf{z}}
\end{array}\right]=\mathbf{0}
$$

For simplicity, let

$$
\mathbf{L} \equiv\left[\begin{array}{ccc}
\mathbf{A}_{x} & \mathbf{0} & \mathbf{0} \\
\mathbf{0} & \mathbf{A}_{y} & \mathbf{0} \\
\mathbf{0} & \mathbf{0} & \mathbf{A}_{z}
\end{array}\right], \quad \mathbf{v} \equiv\left[\begin{array}{c}
\mathbf{v}_{\mathbf{x}} \\
\mathbf{v}_{\mathbf{y}} \\
\mathbf{v}_{\mathbf{z}}
\end{array}\right], \quad \mathbf{b} \equiv\left[\begin{array}{c}
\mathbf{b}_{\mathbf{x}} \\
\mathbf{b}_{\mathbf{y}} \\
\mathbf{b}_{\mathbf{z}}
\end{array}\right]
$$

We use an approach analogous to the Tikhonov regularization [78] to change the problem to finding

$$
\min \left\{\|\mathbf{L} \mathbf{v}-\mathbf{b}\|_{2}^{2}+\lambda^{2}\|\mathbf{D} \mathbf{v}\|_{2}^{2}\right\}
$$

for some positive parameter $\lambda$. After some matrix algebra, one can demonstrate that

$$
\|\mathbf{L} \mathbf{v}-\mathbf{b}\|_{2}^{2}+\lambda^{2}\|\mathbf{D} \mathbf{v}\|_{2}^{2}=\left\|\left[\begin{array}{c}
\mathbf{L} \\
\lambda \mathbf{D}
\end{array}\right] \mathbf{v}-\left[\begin{array}{l}
\mathbf{b} \\
\mathbf{0}
\end{array}\right]\right\|_{2}^{2}
$$

Thus, the constrained problem is equivalent to a least squares problem,

$$
\min \left\{\|\mathbf{L} \mathbf{v}-\mathbf{b}\|_{2}^{2}+\lambda^{2}\|\mathbf{D} \mathbf{v}\|_{2}^{2}\right\} \Longleftrightarrow \min \left\{\left\|\left[\begin{array}{c}
\mathbf{L} \\
\lambda \mathbf{D}
\end{array}\right] \mathbf{v}-\left[\begin{array}{l}
\mathbf{b} \\
\mathbf{0}
\end{array}\right]\right\|_{2}^{2}\right\}
$$

whose solution immediately follows from the normal equations:

$$
\begin{gathered}
{\left[\begin{array}{ll}
\mathbf{L}^{\mathbf{T}} & \lambda \mathbf{D}
\end{array}\right]\left[\begin{array}{c}
\mathbf{L} \\
\lambda \mathbf{D}
\end{array}\right] \mathbf{v}=\left[\begin{array}{ll}
\mathbf{L}^{\mathbf{T}} & \lambda \mathbf{D}
\end{array}\right]\left[\begin{array}{l}
\mathbf{b} \\
\mathbf{0}
\end{array}\right],} \\
\left(\mathbf{L}^{T} \mathbf{L}+\lambda^{2} \mathbf{D}^{T} \mathbf{D}\right) \mathbf{v}=\mathbf{L}^{T} \mathbf{b}
\end{gathered}
$$

\subsubsection{Implementation of velocity field solver}

The velocity field is calculated in two steps. First, from zero velocity as initial guess, the three components are independently estimated from Stokes equation. The same Poisson solver used to calculate pressure is also used in this stage, with the respective components of pressure gradients as the forcing function. Next, this intermediate solution for velocity is used as an initial guess for Equation (44) in order to calculate the incompressible velocity field. The coefficient matrix $\mathbf{L}^{T} \mathbf{L}+\lambda^{2} \mathbf{D}^{T} \mathbf{D}$ is now symmetric, allowing to use the conjugate gradient method [76] as the linear solver. The increased condition number resulting from multiplication by transpose matrices is compensated with preconditioning techniques.

Similar to the pressure field case, the solution for velocity field given by the conjugate 
gradient implementation is analysed by considering the volumetric flux in each direction,

$$
\begin{aligned}
& \Phi_{v_{x}}\left(x_{i}\right)=\iint_{x=x_{i}} v_{x} d y d z, \\
& \Phi_{v_{y}}\left(y_{j}\right)=\iint_{y=y_{j}} v_{y} d x d z, \\
& \Phi_{v_{z}}\left(z_{k}\right)=\iint_{z=z_{k}} v_{z} d x d y,
\end{aligned}
$$

where, given the closed boundaries in $x$ and $y$ directions, the mean value of $\Phi_{v_{x}}(x)$ and $\Phi_{v_{y}}(y)$ are expected to be negligible compared to the mean value of $\Phi_{v_{z}}(z)$. We also assume one standard deviation of $\Phi_{v_{z}}(z)$ divided by its mean value as the numerical uncertainty for the volumetric flux in $z$ direction.

\subsubsection{Permeability and porosity estimation}

Finally, assuming the average flow rate $q_{z}$ through the porous medium as the mean value of $\Phi_{v_{z}}(z)$, we use Darcy's equation to obtain the final value for permeability as

$$
\kappa_{z z}=\frac{q_{z} \mu n_{z} h_{z}}{n_{x} h_{x} n_{y} h_{y}\left(\mathscr{P}_{\text {in }}-\mathscr{P}_{\text {out }}\right)} .
$$

The ratio of the standard deviation to the mean value of $\Phi_{v_{z}}(z)$ is assumed as the relative error for the volumetric flow rate $q_{z}$ and also for the estimated permeability $\kappa_{z z}$. Analogous procedures can be used to calculate $\kappa_{x x}$ and $\kappa_{y y}$ by appropriate choice of open and closed boundaries, although these same closed boundaries prevent meaningful estimates for off-diagonal terms of the permeability tensor.

Porosity from 3D images is simply estimated by counting pore voxels and dividing the result by the total number of voxels.

\subsection{Permeability estimation using finite volume method (FVM)}

In order to compare our results against an established technique, we calculated the absolute permeability of our rock samples using direct simulation of flow by finite volume method. Flow domain discretization was done by converting every pore voxel to a hexahedral computational element, so as to maintain the same computational element used in the proposed FDM. We then used Gmsh [79] and ParaView [80] to visualize the discretized domain. We finally used the OpenFOAM [81] CFD simulator to solve the flow field on the discretized domain. The CFD simulator solves three-dimensional steady state incompressible Navier-Stokes and material balance equations. Knowing the flow field and the pressure gradient across each sample, we calculated the respective absolute permeability.

\subsection{Testing samples}

\subsubsection{Synthetic samples}

We first test the implementation of the minimum divergence method by generating two simple cylindrically symmetric samples: one straight tube (Figure 1a) and a tube 
with changing cross-section (Figure 1b). The cylinder has known analytical solution for velocity profile and volumetric flow [71], while the tube in Figure $1 \mathrm{~b}$ allows to investigate the effect of regularization on achieving small divergence velocity fields.

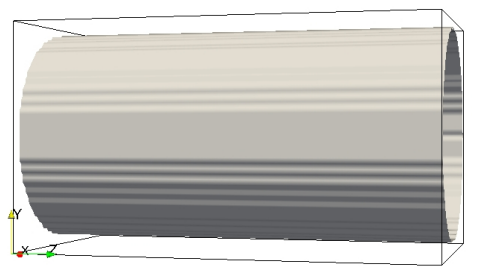

(a)

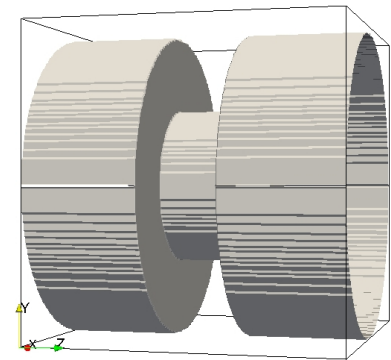

(b)

Figure 1: Cylindrically symmetric samples for initial tests on the finite difference code.

\subsubsection{Benchmarks}

The second group of datasets used to validate the algorithms consists of two established benchmarks. First, a $500^{3}$ Fontainebleau sandstone with voxel length equal to $3 \mu \mathrm{m}$. Details about the image segmentation for this sample can be found in [82]. Second, a $500^{3}$ disordered close pack of non-overlapping equally-sized spheres, digitally generated from Finney's experimental data [3]. Sphere diameter is equal to 100 voxels. The voxel length was chosen to be also $3 \mu \mathrm{m}$. Figure 2 shows these two samples.

\subsubsection{Outcrop samples}

The third group of samples is formed by original $\mu$-CT images for plugs from outcrops of Bentheimer sandstone and Buff Berea sandstone. The two datasets consist of $1000 \times$ $1000 \times 1500$ voxel images. Voxel length for Bentheimer is equal to $5 \mu \mathrm{m}$, while voxel length for Buff Berea is $2 \mu \mathrm{m}$. Figures 3 and 4 show slices of the original 3D $\mu$-CT images. In order to fit the memory available on the used computer nodes, these two samples were divided in twelve $500^{3}$ subsamples, where the corresponding pressure and velocity fields were calculated.

The image segmentation was performed in three steps. First, a $2 \times 2 \times 2$ median filter was applied to reduce the noise level, while trying to preserve pore-grain edges [12]. Second, a procedure called statistical region merging [83] was applied. This procedure starts by defining each voxel as an independent region, and successively performs statistical tests on neighboring regions, merging them if the average intensities are considered similar enough. The algorithm is guided by an user input for the final number of regions. We applied this algorithm recursively, starting with a big number of final regions and reducing the number of clusters until most of the noise was attenuated. Finally, a hard threshold on the merged image was applied to classify each voxel as a pore or a solid phase. 

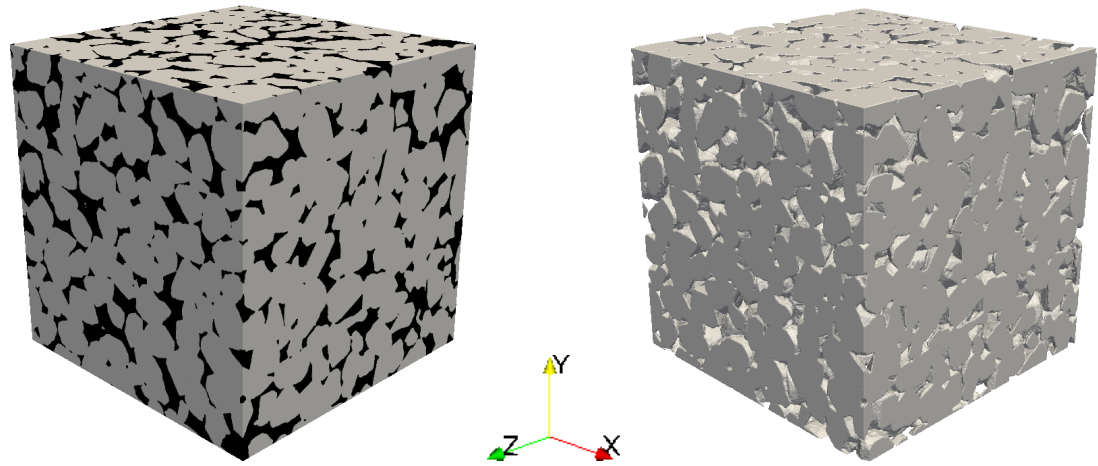

(a) Fontainebleau sandstone
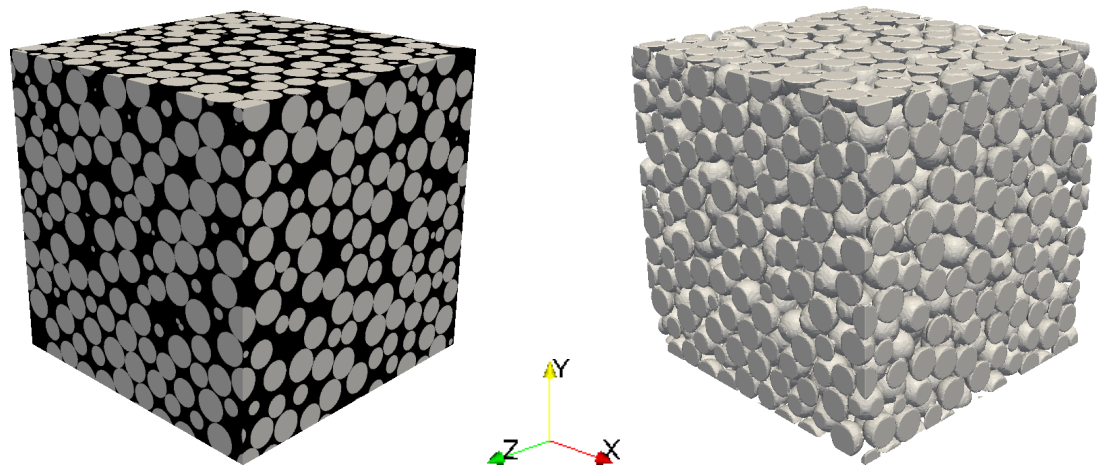

(b) Finney pack

Figure 2: Benchmark samples used for the first test in the finite difference algorithms. Samples have dimensions equal to $500 \times 500 \times 500$ voxels and voxel length equal to $3 \mu \mathrm{m}$. Left: segmented images with black pores and gray grains; right: 3D rendering of pore-grain phase. 


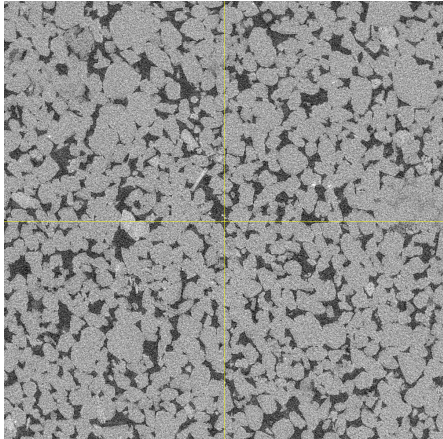

(a) $x y$ slice at $z=750$

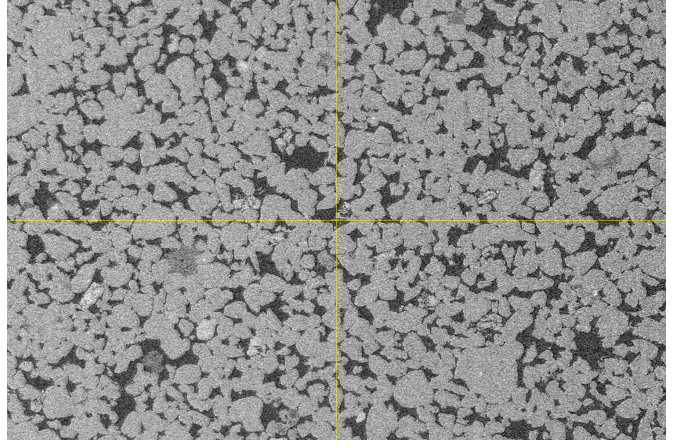

(b) $y z$ slice at $x=500$

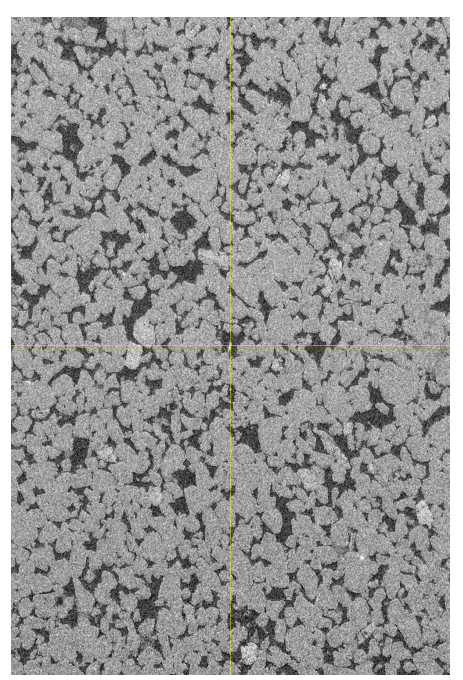

(c) $x z$ slice at $y=500$

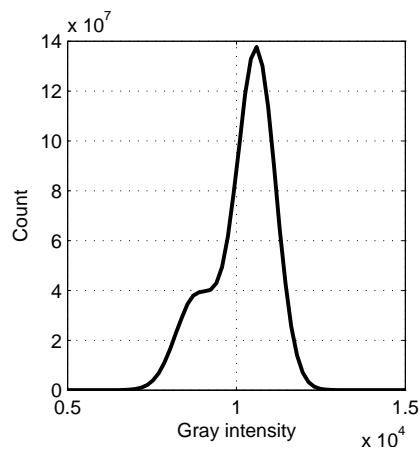

(d) Gray scale histogram

Figure 3: Cross-sections of the Bentheimer sandstone $\mu$-CT image. Voxel length is equal to $5 \mu \mathrm{m}$ and sample dimensions are $1000 \times 1000 \times 1500$ voxels. Coordinates $x, y$ and $z$ are counted in voxels in this figure. 


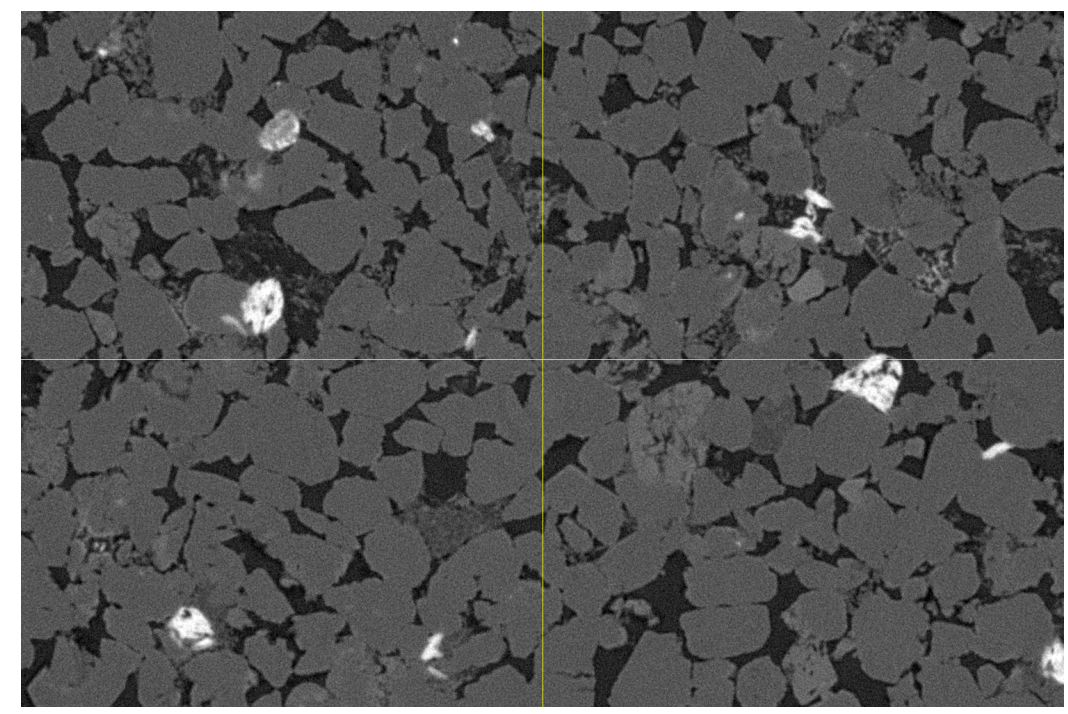

(a) $x y$ slice at $z=500$

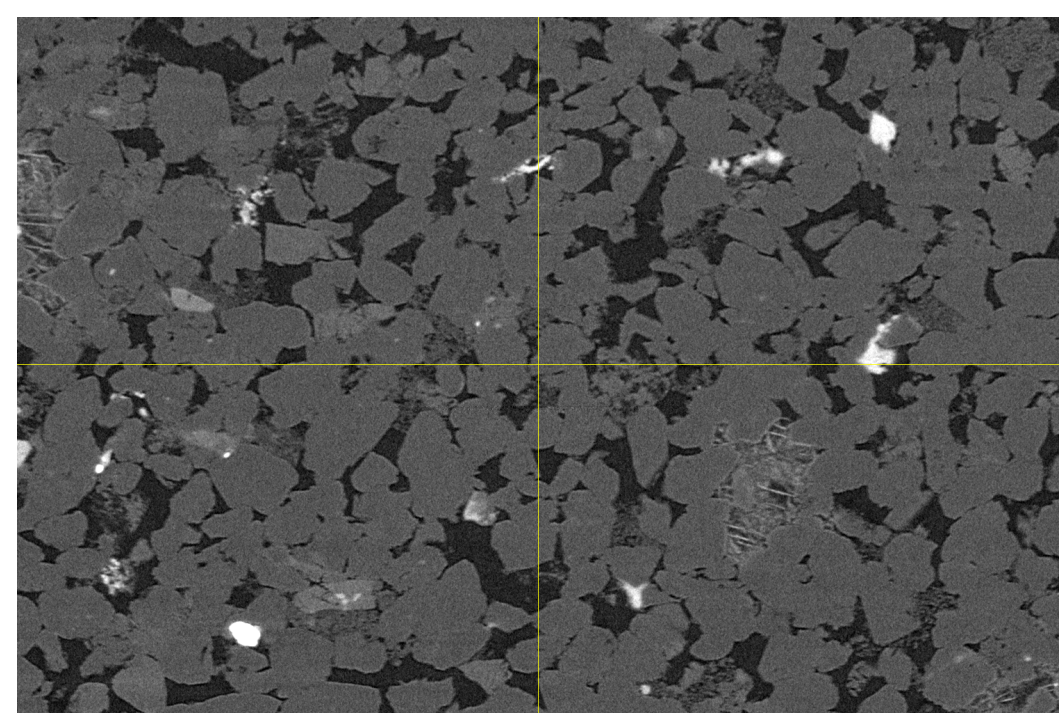

(c) $x z$ slice at $y=500$

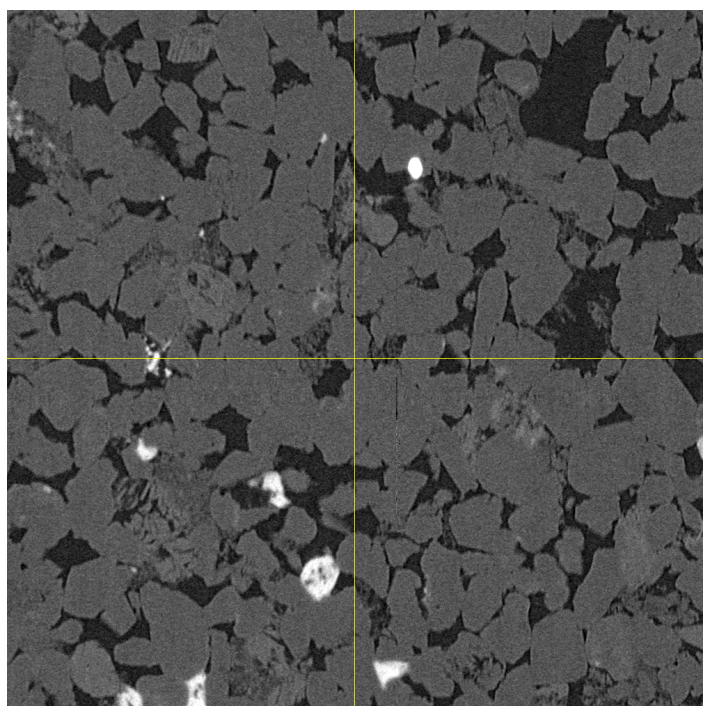

(b) $y z$ slice at $x=750$

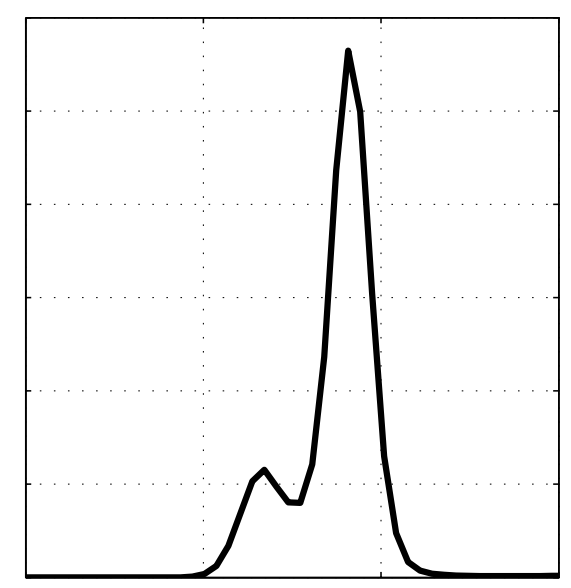

(d) Gray scale histogram

Figure 4: Cross-sections of the Buff Berea sandstone $\mu$-CT image. Voxel length is equal to $2 \mu \mathrm{m}$ and sample dimensions are $1500 \times 1000 \times 1000$ voxels. Coordinates $x, y$ and $z$ are counted in voxels in this figure. 


\subsubsection{Multi-phase fluid distribution for Castlegate sandstone}

As a step towards multi-phase fluid flow, we used the results of two-phase fluid distribution simulation on a Castlegate sandstone, using the LSMPQS method [67]. The method simulates drainage-imbibition curves from capillary dominated two-phase fluid flow, with an initial planar non-wetting phase front trying to invade the pore space fully saturated with the wetting phase. The rock is considered to be perfectly wet by the wetting fluid (zero contact angle).

The simulation goal is to find, for each increasing value of the capillary pressure $p_{c}$, the equilibrium curved interface between the wetting and the non-wetting phases that obeys Young-Laplace equation. On the method formulation, a level set function $\phi(x, y, z, \tau)$, initially at state $\phi(x, y, z, 0)$, evolves with time-like parameter $\tau$ following the partial differential equation

$$
\frac{\partial \phi}{\partial \tau}+F|\vec{\nabla} \phi|=0,
$$

where $F(x, y, z, \tau)$ is the normal component of the interface velocity. To apply this technique to the drainage simulation, the quasi-static movement is governed by

$$
F(x, y, z, \tau)=p_{c}-\gamma C(x, y, z, \tau),
$$

and the equilibrium interface is taken as the zero level set of the solution for Equation (49), i.e. the set of points such that $\phi(x, y, z, \tau)=0$. LSMPQS is implemented using C/FORTRAN computer languages and can be downloaded from the author's web page [84]. The simulation on Castlegate sample [85] generated a set of fourteen fluid configurations for the complete drainage-imbibition process, some of them illustrated in Figure 5 .

Effective permeabilities are calculated for those configurations by running the absolute permeability simulations, described earlier, on each one of the fluid phases. Then, the relative permeability curve is obtained by dividing the effective permeabilities by the absolute permeability of the sample.

\subsection{Computational details}

Our modified finite difference method was implemented using C language and shared memory parallelism through the OpenMP standard [86]. Due to the potentially large amount of memory for big samples, data arrays are stored as a single byte integer per voxel for segmentation data and a single precision floating point number per voxel for pressure and velocity fields.

Further, in order to better use the computer memory available and simulate bigger samples, matrix products in the iterative solvers for pressure and velocity fields are performed by inspecting the segmentation data, without storing the heptadiagonal coefficient matrix. With this implementation, for a $500^{3}$ sample the preconditioned biconjugate gradient stabilized method, used for solving pressure and obtaining initial guess for velocity, requires about $4.2 \mathrm{~GB}$ of computer memory. The preconditioned conjugate gradient method, used to solve Equation (44), although simpler, deals with the three velocity components simultaneously, requiring about $8.4 \mathrm{~GB}$ of computer memory for a $500^{3}$ dataset. We used Jacobi preconditioner in both solvers. 


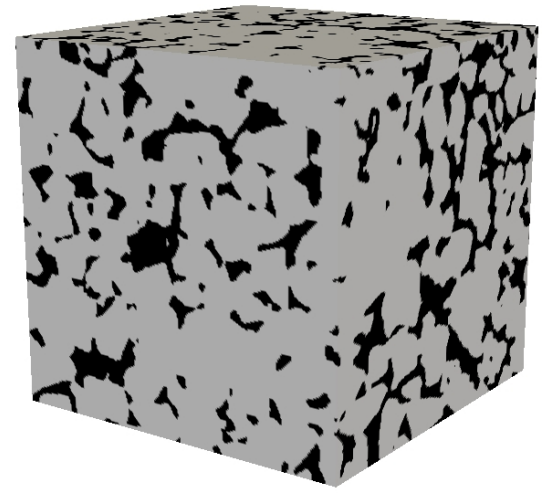

(a) Castlegate dataset

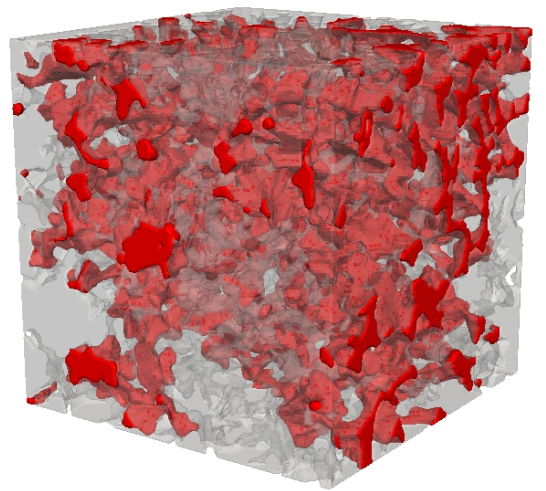

(c) Final drainage stage, $S_{w}=29 \%$

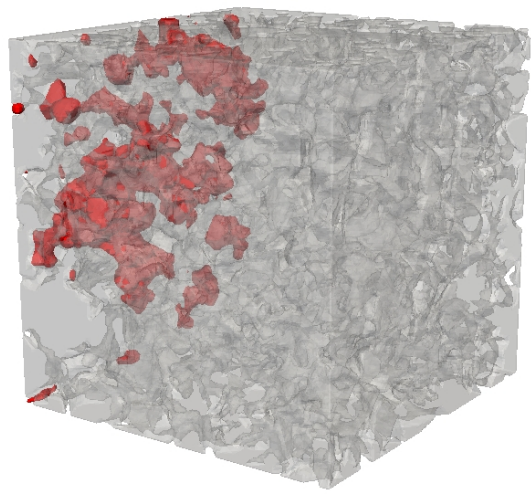

(b) Early drainage stage, $S_{w}=91 \%$

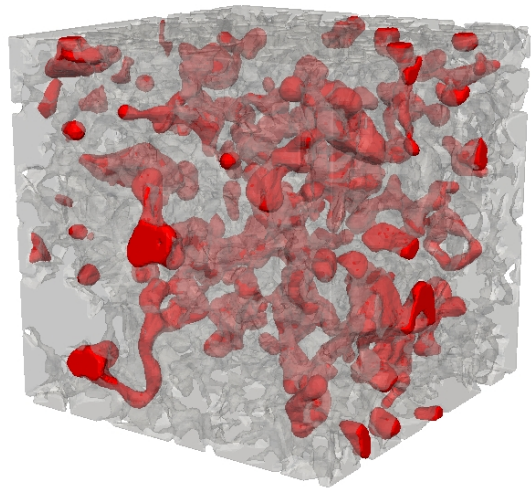

(d) Final imbibition stage, $S_{w}=74 \%$

Figure 5: Simulated drainage-imbibition stages in a $250^{3}$ sample of Castlegate sandstone: (a) grain phase (gray) and pore phase (black); (b) to (d) grain phase is represented by the semi-transparent gray surface and the invading non-wetting phase, colored red, is entering the porous medium through the $x y$ slice at $z=0$ during drainage and returning the same way during imbibition. Approximate values for wetting phase saturation are given by $S_{w}$. Voxel length is equal to $5.6 \mu \mathrm{m}$. 
Table 1: Results for simulation in the cylinder samples. Numbers are presented in consistent arbitrary units. Parentheses denote the numerical uncertainty attributed to the last digit of the respective number. See Figure 6 for velocity profiles.

\begin{tabular}{cccc}
\hline & $q_{x}$ & $q_{y}$ & $q_{z}$ \\
\hline$h_{x, y, z}=2 / 15$ & $9(8) \times 10^{-8}$ & $2(3) \times 10^{-7}$ & $0.125758(8)$ \\
$h_{x, y, z}=1 / 15$ & $3(3) \times 10^{-7}$ & $-2(2) \times 10^{-7}$ & $0.11273(4)$ \\
$h_{x, y, z}=1 / 30$ & $-4(2) \times 10^{-7}$ & $1(6) \times 10^{-8}$ & $0.1031426(6)$ \\
Hagen-Poiseuille & 0 & 0 & $\frac{\pi}{32} \approx 0.0982$ \\
\hline
\end{tabular}

Our calculations were performed using Lonestar Linux high performance system at Texas Advanced Computer Center [87], in a single computer node with two Xeon 5680 series $3.33 \mathrm{GHz}$ hex-core processors (12 cores total) sharing $24 \mathrm{~GB}$ of memory. Simulation times strongly depend on the complexity of the porous medium, varying from a few minutes for samples with $125^{3}$ voxels up to 2 or 3 hours for samples with $500^{3}$ voxels.

\section{Results}

\subsection{Synthetic samples}

Figure 6a shows the results for the velocity field in the cylindrical sample (see Figure 1a) with radius $R=1$, length $L=4$, inlet pressure $\mathscr{P}_{\text {in }}=2$, outlet pressure $\mathscr{P}_{\text {out }}=1$ and fluid viscosity $\mu=1$, using consistent arbitrary units. Pressure field here is omitted since the constant gradient profile used as the initial guess is already the solution for this particular shape [71]. Figure $6 \mathrm{~b}$ compares the $z$-component velocity as a function of the distance from the cylinder axis. The parabolic profile is observed at different resolutions, and tends to the true solution [71]

$$
v_{z}(r)=\frac{\Delta \mathscr{P}}{4 \mu L R^{2}}\left(1-\frac{r^{2}}{R^{2}}\right)=\frac{1}{16}\left(1-r^{2}\right)
$$

as the voxel length decreases. The chosen boundary condition for velocity allows the calculation of the fully developed flow regime. Table 1 shows the measurements for volumetric flow rates and their respective numerical uncertainties, as defined in Section 2.2.8. Flow rates in $x$ and $y$ directions are negligible and the flow rate in $z$ direction approaches the Hagen-Poiseuille equation [71]

$$
q_{z}=\frac{\Delta \mathscr{P} \pi r^{4}}{8 \mu L}=\frac{\pi}{32}
$$

as the voxel length decreases. This simple geometry does not require regularization when calculating the velocity field.

The need for regularization is well appreciated when we look at the results for the pipe with changing cross-section. Figure 7a shows contour levels for the simulated pressure field. Qualitatively, the contour lines terminate perpendicular to the pore-grain interface, showing zero pressure variation normal to the interfaces, as required by the boundary conditions we assumed. Also shown in Figure $7 \mathrm{a}$ is the residual decrease for the linear system of equations as, starting from a pressure field with a constant gradient in $z$ 


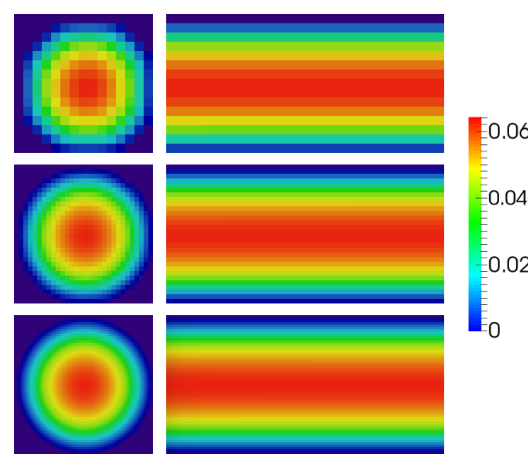

(a) $v_{z}$ field, arbitrary units.

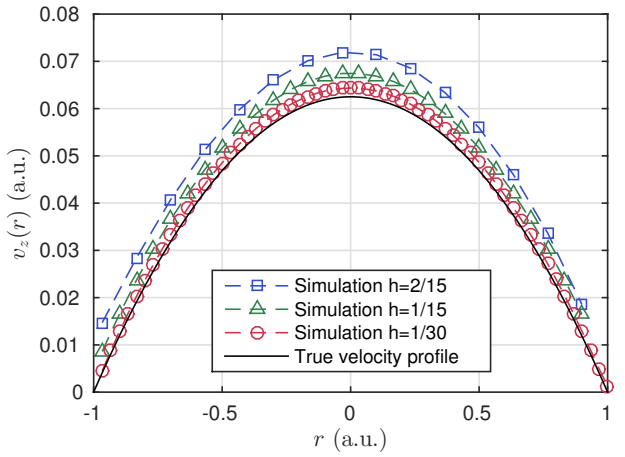

(b) $v_{z}$ profile.

Figure 6: Results for velocity field in the cylinder sample of radius $R=1$ and length $L=4$. (a) $z$-component velocity field in a cross-section (left) and a longitudinal section (right), with voxel lengths $2 / 15$ (top), 1/15 (middle) and 1/30 (bottom); (b) velocity profile as a function of the distance from the cylinder axis. Numbers are shown in consistent arbitrary units (a.u.). See Table 1 for volumetric flux values.

direction, the biconjugate gradient stabilized iterations approach the solution. The flux of the pressure gradient through the sample in $z$ direction (Equation (24)), is shown at the right side of Figure 7a. A relative numerical uncertainty of about $1 \%$ indicates a residual variation from the iterative solver, even after the residual for the pressure Laplace's equation is close to the machine precision.

Figure $7 \mathrm{~b}$ shows the analogous results for the velocity field without regularization. Using $v_{x}=v_{y}=v_{z}=0$ as the initial guess, the residual profile shows that the three velocity components converged to a solution, while the volumetric flux profile for the $z$ direction (Equation (47)), with $40 \%$ of variation, indicates that this solution does not reflect an incompressible flow.

We then use Equation (44) to correct the velocity field, obtaining the results shown by Figure 7c. Although convergence is slower due to the increased condition number of the linear system, the result tends to a velocity field with constant volumetric flux, as required by the incompressible flow regime. Volumetric flux variation is $1.2 \%$ in Figure $7 \mathrm{c}$.

\subsection{Transport properties for Fontainebleau sandstone and Finney pack}

Let us now look in detail at the results of our minimum divergent finite difference method applied on Fontainebleau and Finney samples. We calculated pressure and velocity fields for the $500^{3}$ Fontainebleau in all three directions, by applying the macroscopic pressure gradient in each direction, while selecting appropriate open and closed boundaries. The sphere pack can be generated using arbitrary resolution, and we discretized the same volume using three distinct values for the voxel length, in order to compare the permeability values as a function of resolution.

Figure 8 shows examples of contour levels for the simulated pressure fields and Figure 9 shows $\Phi_{\vec{\nabla} \mathscr{P}}(z)$ profile across these samples, with the respective histogram. It is possible to see some border effects with higher variation of $\Phi_{\vec{\nabla} \mathscr{P}}(z)$, which would enforce higher 

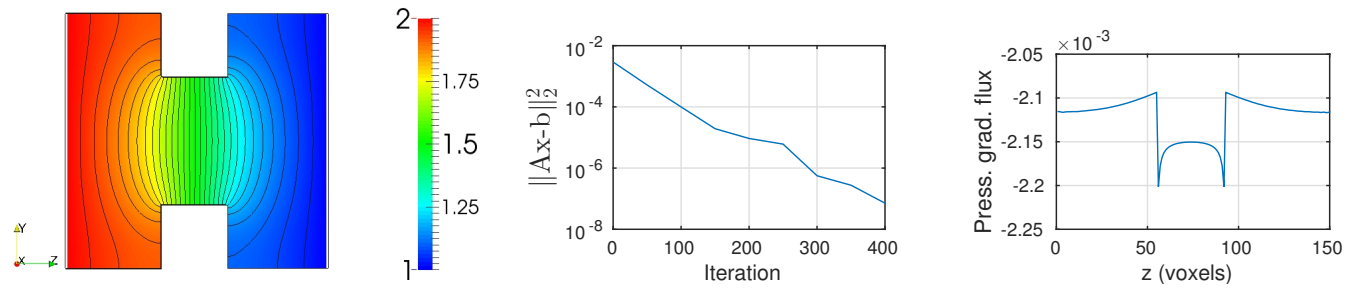

(a) Left: pressure field profile in a $z y$ section; middle: residual for the linear solver implementation; right: flux of the pressure gradient through the sample in z direction.
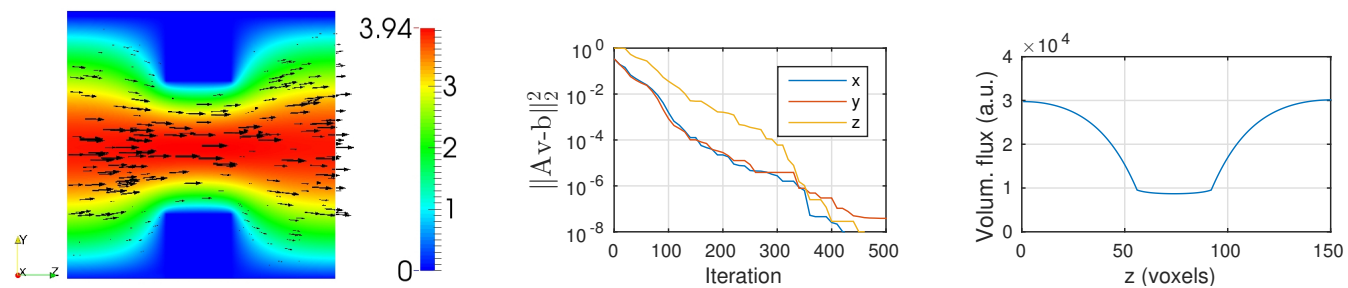

(b) Left: velocity field profile, before regularization, in a $z y$ section; middle: residual for the linear solver implementation; right: volumetric flux through the sample in $\mathrm{z}$ direction.
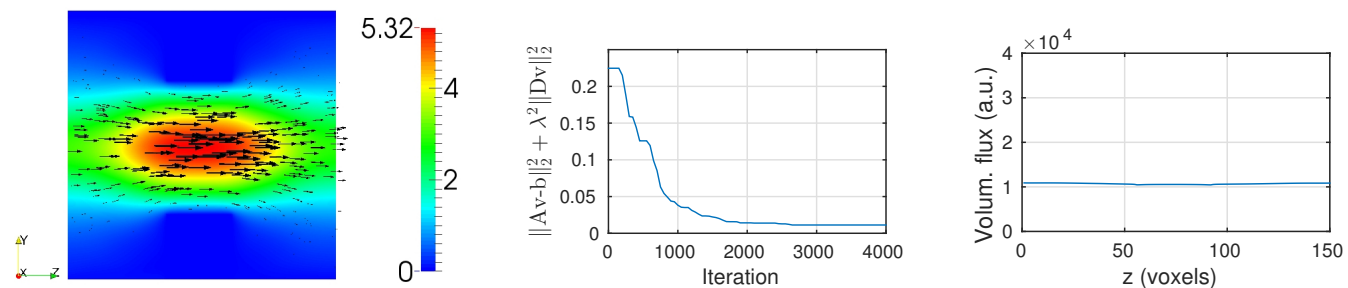

(c) Left: velocity field profile, after regularization, in a $z y$ section; middle: residual for the linear solver implementation; right: volumetric flux through the sample in z direction.

Figure 7: Simulation results for the pipe with changing cross-section. 

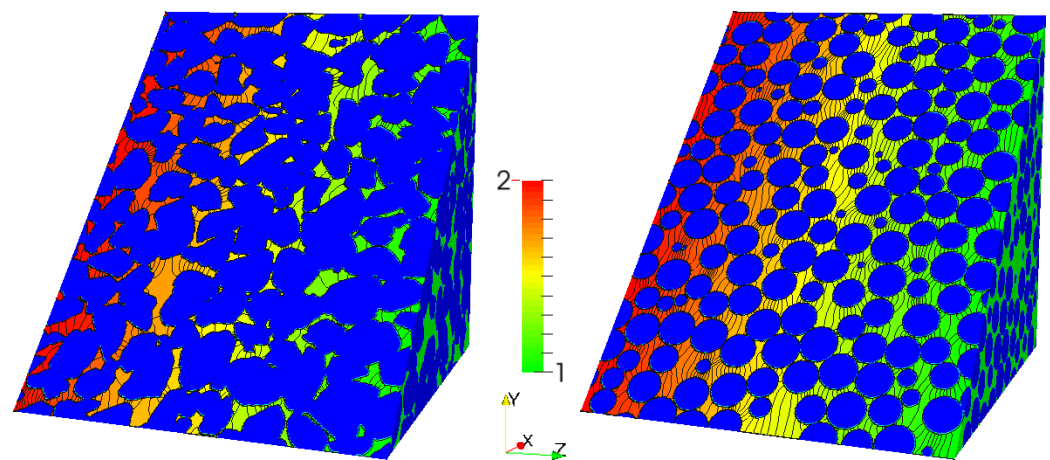

Figure 8: Pressure contour levels for Fontainebleau (left) and Finney (right) samples. Numbers are presented in arbitrary units.

Table 2: Results for simulation on Fontainebleau and Finney datasets. Permeability values are in Darcy.

\begin{tabular}{cccccc}
\hline Sample & Porosity & $\Delta \Phi_{\vec{\nabla} p}$ & $\kappa$ FDM & $\kappa$ FVM & $\kappa$ literature \\
\hline \multirow{2}{*}{ Fontainebleau } & 0.1933 & $\pm 6.0 \%$ & $\kappa_{x x}=2.27( \pm 7 \%)$ & & \\
& & $\pm 2.8 \%$ & $\kappa_{y y}=2.22( \pm 4 \%)$ & - & $1-3$ \\
\hline Finney $3 \mu \mathrm{m}$ & 0.3541 & $\pm 2.2 \%$ & $\kappa_{z z}=49.50( \pm 5.4 \%)$ & 41.5 & \\
Finney $6 \mu \mathrm{m}$ & 0.3485 & $\pm 2.1 \%$ & $\kappa_{z z}=35.47( \pm 1.6 \%)$ & 43.5 & $42-58$ \\
Finney $12 \mu \mathrm{m}$ & 0.3367 & $\pm 2.4 \%$ & $\kappa_{z z}=46.06( \pm 2.3 \%)$ & 42.8 & \\
\hline
\end{tabular}

volumetric fluid flow at the open boundaries. This, in turn, is later controlled by the regularization procedure. The relative numerical uncertainty for the pressure field is shown in Table 2.

Figure 10 shows streamlines for representative Fontainebleau and Finney velocity fields. The residual variations for the volumetric flux are shown in Figure 11, while Table 2 details the final values obtained for absolute permeability.

Results for absolute permeability can be compared to data in literature. Permeability for disordered mono-sized sphere pack and clean sandstones has been accurately described by the Kozeny-Carman equation [2, 88],

$$
\kappa=\frac{D_{g}^{2} \phi^{3}}{36 \tau(1-\phi)^{2}},
$$

where $D_{g}$ is the grain diameter and $\tau$ is the Kozeny constant, equal to 5 for the sphere pack. Mavko and Nur [88] showed that the introduction of the percolation threshold porosity $\phi_{c}$ (the minimum porosity value to provide a connected path through the sample) in Kozeny-Carman model as

$$
\kappa=\frac{D_{g}^{2}\left(\phi-\phi_{c}^{3}\right)}{36 \tau\left(1-\phi+\phi_{c}\right)^{2}},
$$

results in a substantial improvement in quantitative fit for clean and well-sorted rocks, and experimentally determined permeability-particle diameter relations for fused glass 


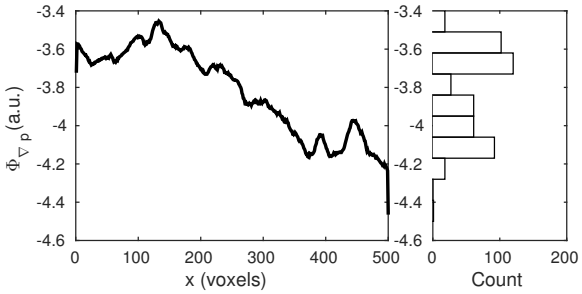

(a) Fontainebleau, flow in $x$ direction

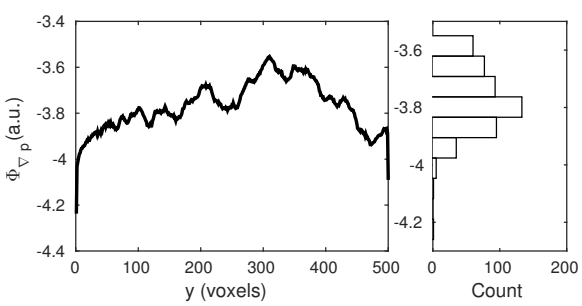

(c) Fontainebleau, flow in $y$ direction

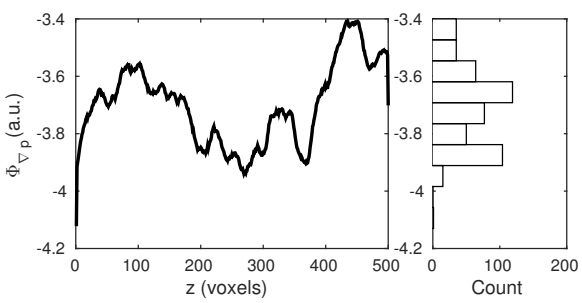

(e) Fontainebleau, flow in $z$ direction

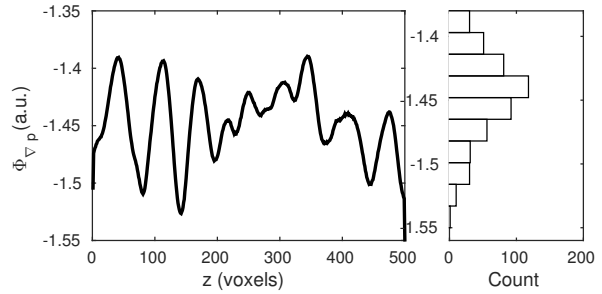

(b) Finney, voxel length $3 \mu \mathrm{m}$

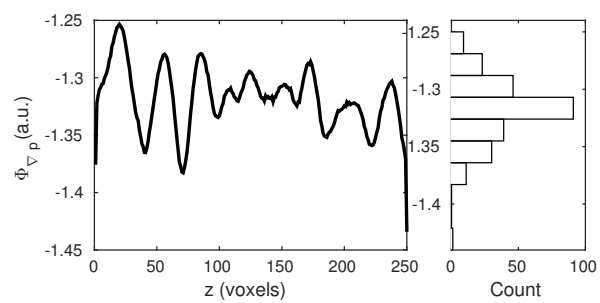

(d) Finney, voxel length $6 \mu \mathrm{m}$

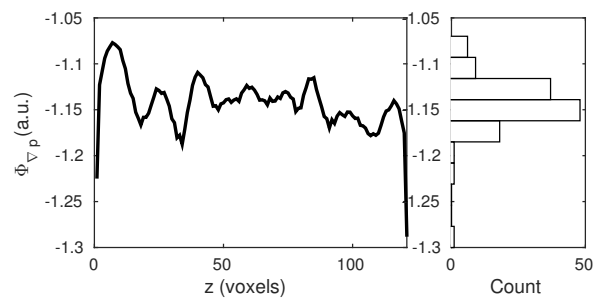

(f) Finney, voxel length $12 \mu \mathrm{m}$

Figure 9: Profiles for the flux of pressure gradient, showing the residual variations for Fontainebleau simulations in different directions and Finney simulations with different voxel lengths. The plots are presented in consistent arbitrary units (a.u.) for simplicity, since the main measure here is the relative variation $\Delta \Phi_{\vec{\nabla} \mathscr{P}}$. Permeability is calculated using the macroscopic pressure gradient in consistent units.
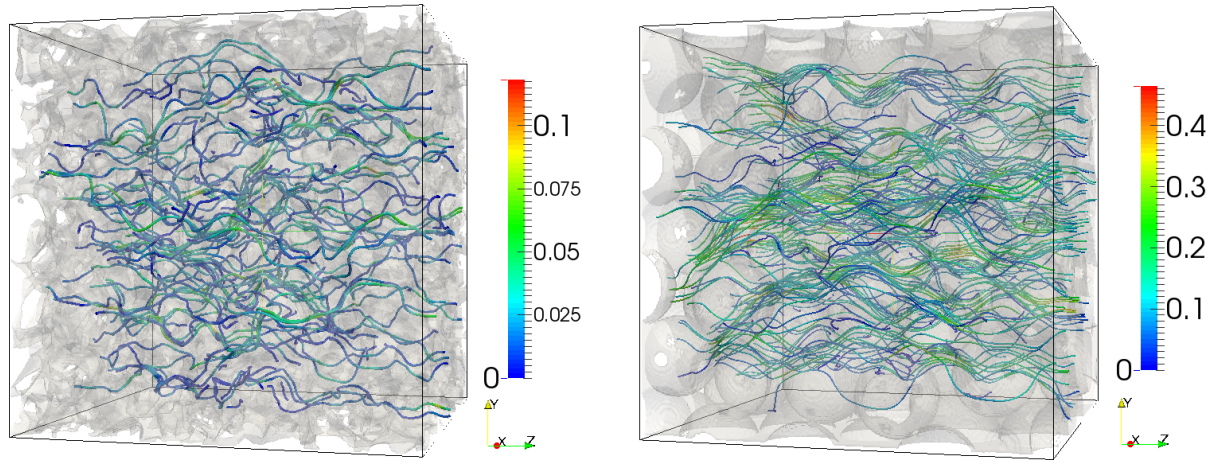

Figure 10: Streamlines for representative velocity fields in Fontainebleau and Finney simulations. 


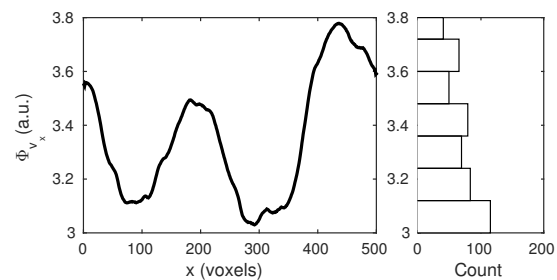

(a) Fontainebleau, flow in $x$ direction

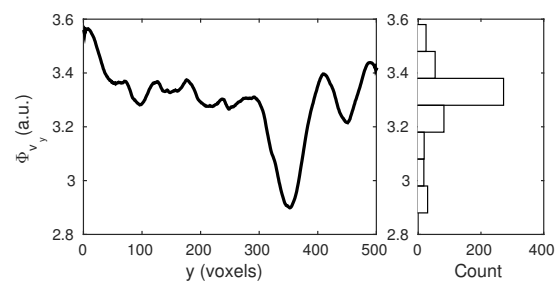

(c) Fontainebleau, flow in $y$ direction

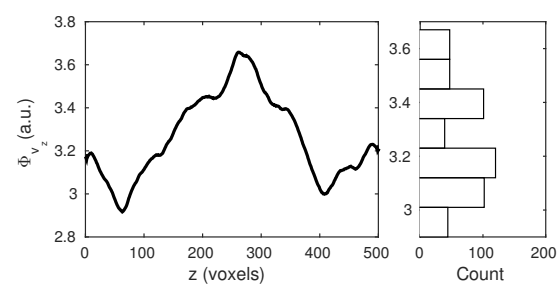

(e) Fontainebleau, flow in $z$ direction

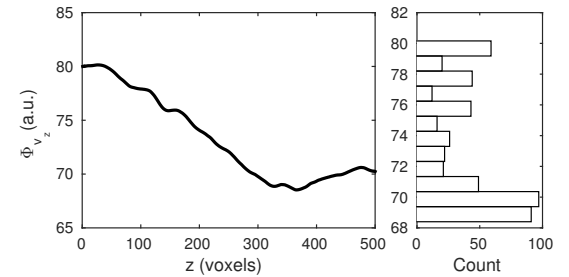

(b) Finney, voxel length $3 \mu \mathrm{m}$

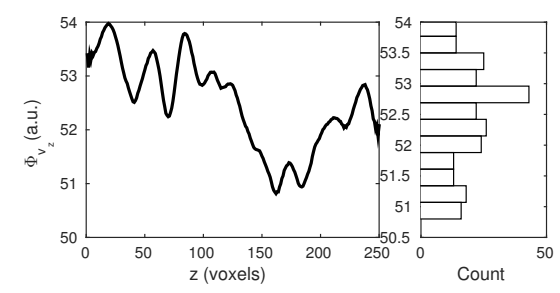

(d) Finney, voxel length $6 \mu \mathrm{m}$

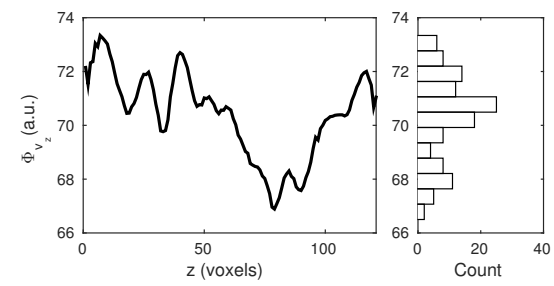

(f) Finney, voxel length $12 \mu \mathrm{m}$

Figure 11: Profiles for volumetric flow rates across the samples for Fontainebleau simulations in different directions and Finney simulations with different voxel lengths. The final value for permeability is given in Table 2. 
beads and Fontainebleau samples. Permeability for sister samples of the Fontainebleau sandstone used in our study is reported by Shabro et al. [69]. In table 2 we report the range of permeabilities using Equation 53 and data from literature [88] for the sphere pack, and the range of permeabilities using data from literature [69, 88]. We see good agreement among the calculated values and the expected ones. The close values for Fontainebleau permeability in different directions shows a fairly isotropic sample, and FVM shows to be less affected by the voxel length in the sphere pack sample.

Our choice for the regularization parameter is illustrated in Figure 12, where the $L_{2}$ norm of the absolute residual for the Stokes problem, the $L_{2}$ norm of the absolute residual for the divergence problem, and the absolute permeability are plotted as a function of $\lambda$. The figure also shows the equivalent of the so-called $L$-curve, for which the maximum curvature point is a common choice for $\lambda$ when using Tikhonov regularization in ill-posed problems [78]. Our method aims for a low divergence solution, and for this reason the value of $\lambda$ is increased until the permeability approaches its asymptotic value.

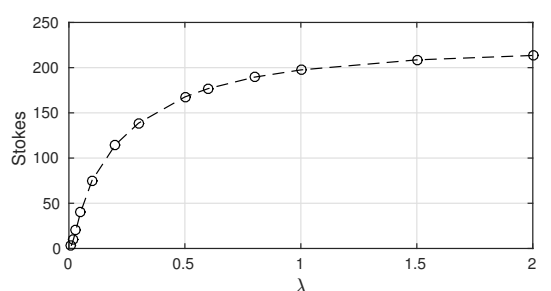

(a)

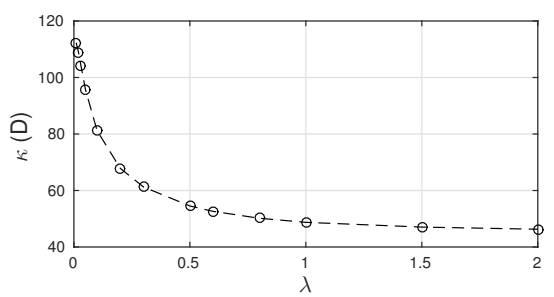

(c)

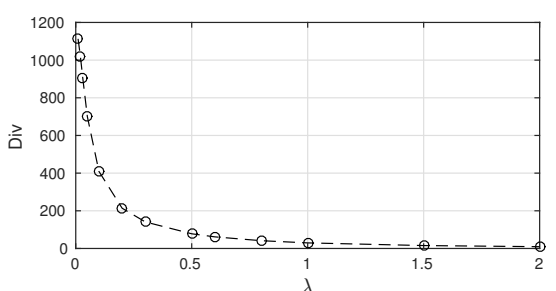

(b)

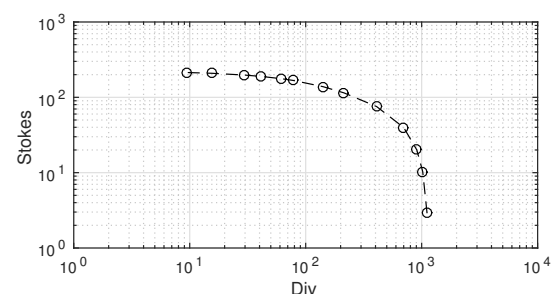

(d)

Figure 12: (a) Stokes residual $\|\mathbf{L v}-\mathbf{b}\|_{2}$, (b) divergence residual $\|\mathbf{D v}\|_{2}$, and (c) absolute permeability $\kappa$ as a function of the regularization parameter $\lambda$, for the $125^{3}$ sphere pack. The cross plot of Stokes and divergence residuals is shown in (d). The choice for the regularization parameter in this particular problem is $\lambda=2$.

\subsection{Permeability for Bentheimer and Buff Berea sandstones}

Representative slices for the segmentation results are shown in Figure 13. The gray scale histograms show that the strategy of a median filter followed by the statistical region merging was sufficient as a preparation for thresholding in these good quality images.

Figure 14 compares the results for absolute permeability using the finite difference method, finite volume method, and laboratory measurements on sister samples through 

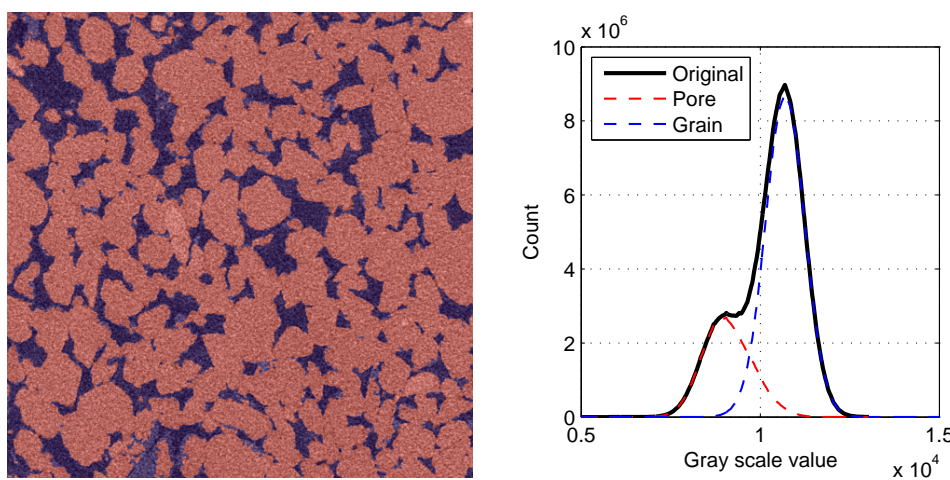

(a) Bentheimer
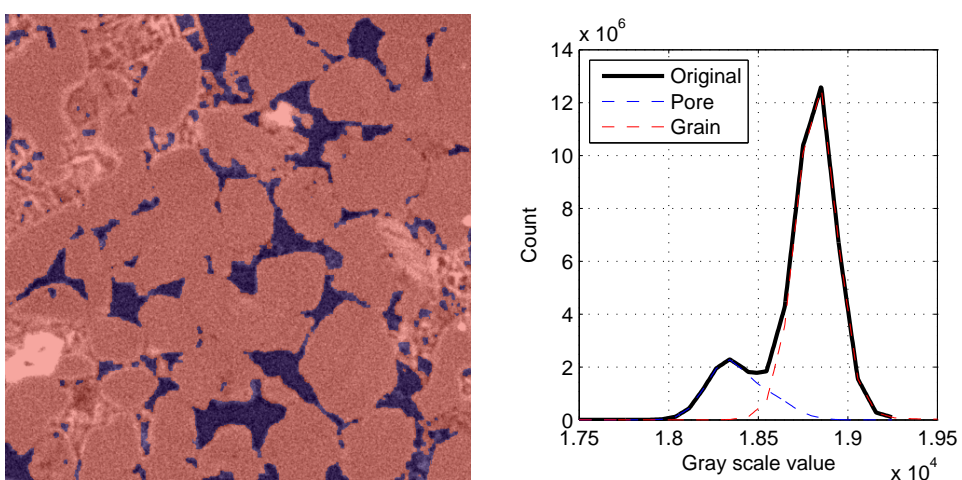

(b) Buff Berea

Figure 13: Example of segmentation results for the two outcrop samples. Blue represents pore space and red represents solid space. Slices' sizes are $500 \times 500$ voxels. 

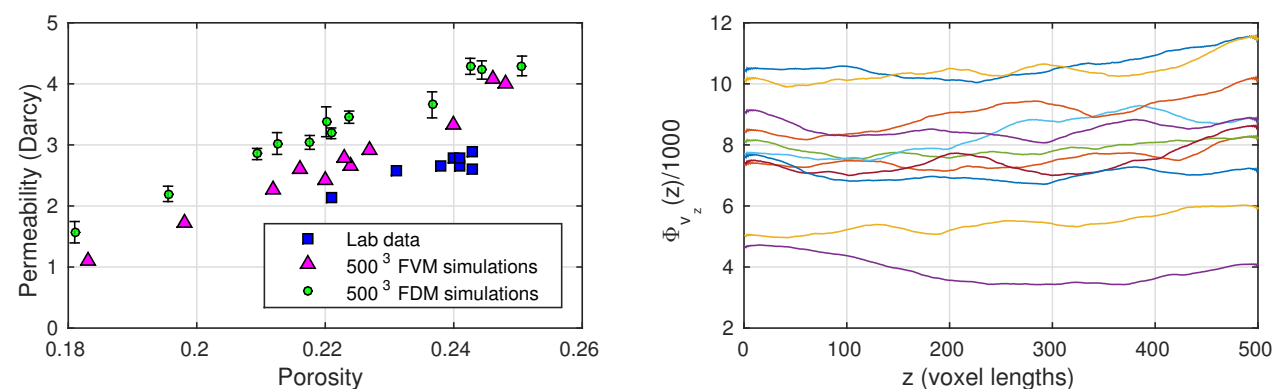

(a) Bentheimer sandstone
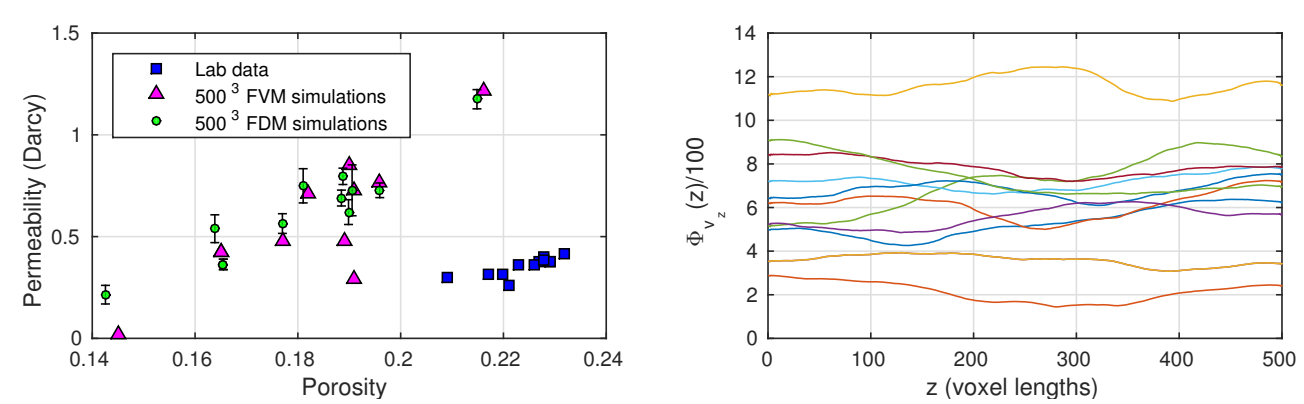

(b) Buff Berea sandstone

Figure 14: Results for absolute permeability from the finite difference method (FDM), finite volume method (FVM) and full plug laboratory data (left) and the volumetric flux profile from FDM (right). Porosity for FVM is calculated from the final meshed volume of the pore space, while the reported porosity for FDM is the number of pore voxels after segmentation divided by the total number of voxels. Laboratory samples have cylindrical shapes with diameter of $3.8 \mathrm{~cm}$ and length between 4 and $9 \mathrm{~cm}$. The error bars for FDM on the left correspond to a relative error equal to one standard deviation of $\Phi_{v_{z}}(z)$ divided by its mean value.

routine core analysis. We again see close agreement among the results from the two numerical methods, with FDM permeabilities slightly higher than the FVM results. Both methods calculate higher values for the permeability of the $500^{3}$ subsets when compared to the experimental results from core data.

The better comparison for Bentheimer sandstone simulations and experimental results reflects the reasonable homogeneity of the sample, as well as its small grain size, making a $500^{3}$ subsample, which comprehends a volume of $(2.5 \mathrm{~mm})^{3}$, representative of the macroscopic medium. In the Buff Berea sandstone, permeability from the digital subsets is higher than the laboratory measurements on the full plugs, indicating that these subsets may not represent the average properties of the rock.

\subsection{Two-phase fluid displacement in Castlegate sandstone}

Figure 15 shows the results for relative permeability, calculated by applying the minimum divergence finite difference method to the drainage simulations for Castlegate sandstone. Permeability was calculated in the direction of the bulk non-wetting phase movement. The points show the trend normally observed in experimental data, with smaller 


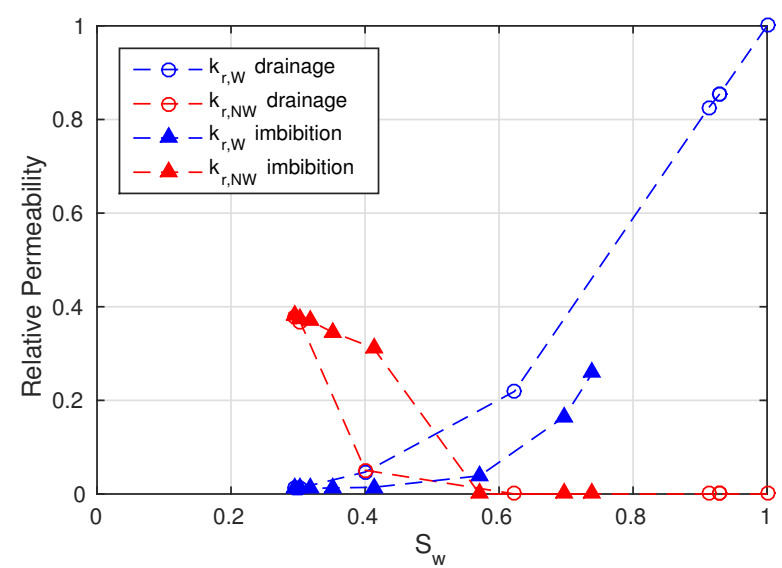

Figure 15: Results for relative permeability on the LSMPQS drainage simulation, by applying the finite difference method.

end point relative permeability for the wetting phase, as a result of blocking of flow paths by the trapped non-wetting phase blobs at the center of the larger pores (see Figure $5 \mathrm{~d}$ ).

The use of LSMPQS for drainage-imbibition simulations together with our regularized finite differences has some advantages over other fluid flow simulations. First, the only major parameter to be set is the regularization constant $\lambda$ on Equation (44). Everything else can be automatically set based on the sample geometry and the pore-grain distribution. Furthermore, an automated procedure to determine optimum $\lambda$ could be developed in the future. The main disadvantage of finite volume in this case is the need to update the mesh for every drainage-imbibition step, resulting in a time consuming pre-processing.

In the absence of enough computational power to carry the dynamic two phase flow simulations, treating one phase at a time in images like the ones in Figure 15, as it was the only fluid present in pore space, has been a common method in literature to estimate relative permeability curves $[44,74,89]$. However, we emphasize that this approach is used here as a preliminary step towards using FDM for multiphase fluid flow simulation. An extension of our regularized finite difference method to the real two-phase fluid flow would be based on handling boundary conditions for pressure and properly coupling velocity from each fluid phase at fluid-fluid interface. This modification would preserve the main advantages of finite differences: the use of segmented images without the need of any re-meshing and the computational efficiency brought by the conjugate gradient family of linear solvers. Such extension is left for future work.

\section{Conclusion}

We developed and validated a novel modification to finite difference discretization of Stokes equation. Tikhonov regularization showed to be a reliable tool for maintaining low divergence in the solution of Stokes equation with zero Neumann open flow boundary conditions. This constitutes an alternative and effective way to use finite difference 
method to calculate velocity field in fully developed regime, and as well estimate the hydraulic permeability without additional simplifications or forced boundary conditions.

We assessed the quality of the results based on over 50 simulations using four distinct sandstone images plus one sphere pack. Our simulations showed good agreement with the ones from the finite volume method, which is an established technique for solving the Stokes equation in incompressible flow regime. We could also get good comparisons between the calculations in small subsets and experimental results for sphere packs, Fontainebleau sandstone and Bentheimer sandstone. Values for Buff Berea sandstones are affected by the more complicated pore geometry on these samples, and simulations using images from larger volumes are needed.

Relative permeability curves for Castlegate sandstone show the expected behavior in a drainage-imbibition cycle.

The main advantage of our enhanced finite difference method over the finite volume in porous medium applications is the direct use of the voxelized medium, as opposed to the time consuming mesh pre-processing usually needed in the finite volume technique. The alternative of using a voxelized finite volume mesh requires large amounts of memory.

Besides having a good estimate of the porous medium effective properties, the finite difference method also generates a detailed description of pressure and velocity fields. This information can be used in the future to investigate the relation between the pore space and the macroscopic property in complex rocks, where simplified models are not available.

We observed one main limitation in the regularized FDM. Poor resolved pore throats in complex geometries can generate anomalous values for gradient fields at the open boundaries and inhibit proper convergence for the pressure and velocity fields. The consequent anomalous inlet and outlet volumetric flows have to be compensated by increasing the regularization parameter, leading to higher residuals for the Stokes equations.

With the assumption of capillary dominated fluid flow, each phase flows through a separate network of channels determined by the initial fluid distribution. We estimated effective permeability by assigning the voxels corresponding to one fluid phase to grain voxels and letting the other fluid phase flow in the remaining space. This approach has the advantage of using the same code designed to calculate absolute permeability, but also has the limitation of imposing zero velocity at the fluid-fluid interfaces.

In future work, we plan to extend the boundary conditions to properly handle fluidfluid interfaces in multi-phase flow simulations, while addressing the aforementioned open boundary problem and developing an automated procedure for estimating the regularization parameter. Finally, open boundaries parallel to the macroscopic pressure gradient can be tested for volume calculating the full permeability tensor.

\section{Acknowledgments}

R.V. has been supported by Petrobras. M.M. has been in part supported by American Chemical Society Petroleum Research Fund (grant \#52340-DNI9) and M.P. has been in part supported by NSF EAR CAREER \#1255622.

The authors thank Petrobras Research Center for providing the X-ray microtomography datasets and respective routine core analysis on Bentheimer and Buff Berea sandstones. We used the image processing package Fiji [90] to perform the image segmentation on these samples and also generate Figures 3, 4, and 13. 
Velocity field visualization in Figure 7 and all the 3D visualization figures were generated using the open source application ParaView [91].

The authors acknowledge the Texas Advanced Computing Center (TACC) [87] at The University of Texas at Austin for providing high performance computational resources that have contributed to the research results reported within this paper.

\section{References}

[1] G. E. Archie, The electrical resistivity log as an aid in determining some reservoir characteristics, Trans. AIMe 146 (99) (1942) 54-62.

[2] E. J. Peters, Advanced Petrophysics: geology, porosity, absolute permeability, heterogeneity, and geostatistics, Vol. 1, Greenleaf Book Group, 2012.

[3] J. Finney, Random packings and the structure of simple liquids. I. The geometry of random close packing, Proceedings of the Royal Society of London. A. Mathematical and Physical Sciences 319 (1539) (1970) 479-493.

[4] M. H. Ellis, M. C. Sinha, T. A. Minshull, J. Sothcott, A. I. Best, An anisotropic model for the electrical resistivity of two-phase geologic materials, Geophysics 75 (6) (2010) E161-E170.

[5] G. Jin, C. Torres-Verdín, E. Toumelin, Comparison of NMR simulations of porous media derived from analytical and voxelized representations, Journal of Magnetic Resonance 200 (2) (2009) 313320 .

[6] S. Bryant, N. Pallatt, Predicting formation factor and resistivity index in simple sandstones, Journal of Petroleum Science and Engineering 15 (2) (1996) 169-179.

[7] M. J. Blunt, B. Bijeljic, H. Dong, O. Gharbi, S. Iglauer, P. Mostaghimi, A. Paluszny, C. Pentland, Pore-scale imaging and modelling, Advances in Water Resources 51 (2013) 197-216.

[8] D. Wildenschild, A. P. Sheppard, X-ray imaging and analysis techniques for quantifying pore-scale structure and processes in subsurface porous medium systems, Advances in Water Resources 51 (2013) 217-246.

[9] B. P. Flannery, H. W. Deckman, W. G. Roberge, K. L. D'AMICO, Three-dimensional X-ray microtomography, Science 237 (4821) (1987) 1439-1444.

[10] V. Cnudde, M. Boone, High-resolution X-ray computed tomography in geosciences: a review of the current technology and applications, Earth-Science Reviews 123 (2013) 1-17.

[11] P. Iassonov, T. Gebrenegus, M. Tuller, Segmentation of X-ray computed tomography images of porous materials: a crucial step for characterization and quantitative analysis of pore structures, Water resources research $45(9)$.

[12] S. Schlüter, A. Sheppard, K. Brown, D. Wildenschild, Image processing of multiphase images obtained via X-ray microtomography: a review, Water Resources Research 50 (4) (2014) 36153639. doi:10.1002/2014WR015256. URL http://dx.doi.org/10.1002/2014WR015256

[13] X. Zhan, L. M. Schwartz, M. N. Toksöz, W. C. Smith, F. D. Morgan, Pore-scale modeling of electrical and fluid transport in berea sandstone, Geophysics 75 (5) (2010) F135-F142.

[14] S. Yanici, J.-Y. Arns, Y. Cinar, W. Pinczewski, C. Arns, Percolation effects of grain contacts in partially saturated sandstones: deviations from Archie's Law, Transport in porous media 96 (3) (2013) 457-467.

[15] C. Arns, F. Bauget, A. Limaye, A. Sakellariou, T. Senden, A. Sheppard, R. M. Sok, V. Pinczewski, S. Bakke, L. I. Berge, P. E. Oren, M. A. Knackstedt, Pore scale characterization of carbonates using X-ray microtomography, Spe Journal 10 (4) (2005) 475-484.

[16] M. Knackstedt, C. Arns, A. Sheppard, T. Senden, R. Sok, Y. Cinar, A. Olafuyi, W. Pinczewski, G. Padhy, M. Ioannidis, Pore scale analysis of electrical resistivity in complex core material, in: International Symposium of the Society of Core Analysts held in Calgary, Canada, 2007, pp. 10-12.

[17] M. A. Knackstedt, S. Latham, M. Madadi, A. Sheppard, T. Varslot, C. Arns, Digital rock physics: $3 \mathrm{D}$ imaging of core material and correlations to acoustic and flow properties, The Leading Edge 28 (1) (2009) 28-33.

[18] H. Andrä, N. Combaret, J. Dvorkin, E. Glatt, J. Han, M. Kabel, Y. Keehm, F. Krzikalla, M. Lee, C. Madonna, M. Marsh, T. Mukerji, E. H. Saenger, R. Sain, N. Saxena, S. Ricker, A. Wiegmann, X. Zhan, Digital Rock Physics Benchmarks-Part II: Computing effective properties, Computers \& Geosciences 50 (2013) 33-43. 
[19] W. Yue, G. Tao, D. Liu, W. Yang, Numerical simulation of non-archie electrophysical property of saturated rock with lattice boltzmann method, Petroleum Science 6 (1) (2009) 24-28.

[20] J. Dvorkin, N. Derzhi, E. Diaz, Q. Fang, Relevance of computational rock physics, Geophysics 76 (5) (2011) E141-E153.

[21] C. H. Arns, M. A. Knackstedt, W. V. Pinczewski, E. J. Garboczi, Computation of linear elastic properties from microtomographic images: Methodology and agreement between theory and experiment, Geophysics 67 (5) (2002) 1396-1405.

[22] C. Arns, An analysis of NMR-permeability scaling rules by numerical MRI, in: 48th Annual Logging Symposium, Society of Petrophysicists and Well-Log Analysts, 2007.

[23] C. Arns, A. P. Sheppard, R. M. Sok, M. Knackstedt, NMR petrophysical predictions on digitized core images, Petrophysics 48 (3) (2007) 202.

[24] C. Arns, Y. Meleán, Accurate simulation of NMR responses of mono-mineralic carbonate rocks using Xray-CT Images, in: SPWLA 50th Annual Logging Symposium, 2009.

[25] E. Toumelin, Pore-Scale Petrophysical Models for the Simulation and Combined Interpretation of Nuclear Magnetic Resonance and Wide-Band Electromagnetic Measurements of Saturated Rocks, The University of Texas at Austin, 2006.

URL http://www.pge.utexas.edu/images/pdfs/theses06/toumelin.pdf

[26] S. Bryant, M. Blunt, Prediction of relative permeability in simple porous media, Physical Review A 46 (1992) 2004-2011. doi:10.1103/PhysRevA.46.2004. URL http://link.aps.org/doi/10.1103/PhysRevA.46.2004

[27] S. L. Bryant, P. R. King, D. W. Mellor, Network model evaluation of permeability and spatial correlation in a real random sphere packing, Transport in Porous Media 11 (1) (1993) 53-70.

[28] M. J. Blunt, Flow in porous media-pore-network models and multiphase flow, Current opinion in colloid \& interface science 6 (3) (2001) 197-207.

[29] M. J. Blunt, M. D. Jackson, M. Piri, P. H. Valvatne, Detailed physics, predictive capabilities and macroscopic consequences for pore-network models of multiphase flow, Advances in Water Resources 25 (8) (2002) 1069-1089.

[30] M. A. Mousavi, S. L. Bryant, Connectivity of pore space as a control on two-phase flow properties of tight-gas sandstones, Transport in porous media 94 (2) (2012) 537-554.

[31] A. Mehmani, M. Prodanović, The effect of microporosity on transport properties in porous media, Advances in Water Resources 63 (2014) 104-119.

[32] A. Mehmani, M. Prodanović, F. Javadpour, Multiscale, multiphysics network modeling of shale matrix gas flows, Transport in porous media 99 (2) (2013) 377-390

[33] V. Shabro, C. Torres-Verdn, F. Javadpour, K. Sepehrnoori, Finite-difference approximation for fluid-flow simulation and calculation of permeability in porous media, Transport in Porous Media 94 (2012) 775-793

[34] A. J. Ladd, Numerical simulations of particulate suspensions via a discretized Boltzmann equation. Part 1. Theoretical foundation, Journal of Fluid Mechanics 271 (1994) 285-309.

[35] A. J. Ladd, Numerical simulations of particulate suspensions via a discretized Boltzmann equation Part 2. Numerical results, Journal of Fluid Mechanics 271 (1994) 311-339.

[36] C. H. Arns, M. A. Knackstedt, W. V. Pinczewski, N. S. Martys, Virtual permeametry on microtomographic images, Journal of Petroleum Science and Engineering 45 (1) (2004) 41-46.

[37] J. Peiró, S. Sherwin, Finite difference, finite element and finite volume methods for partial differential equations, in: Handbook of materials modeling, Springer, 2005, pp. 2415-2446.

[38] N. Moshkin, D. Yambangwai, On numerical solution of the incompressible navier-stokes equations with static or total pressure specified on boundaries, Mathematical Problems in Engineering 2009

[39] L. T. Akanji, S. K. Matthai, Finite Element-Based Characterization of Pore-Scale Geometry and Its Impact on Fluid Flow, Transport in Porous Media 81 (2) (2010) 241-259. doi:10.1007/ s11242-009-9400-7. URL http://link.springer.com/10.1007/s11242-009-9400-7

[40] L. Falco, J. Wegner, L. Ganzer, M. Qi, Pore-Scale Simulation of Viscoelastic Polymer Flow using a Stabilised Finite Element Method, Society of Petroleum Engineers, 2013. doi:10.2118/165987-MS. URL http://www.onepetro.org/doi/10.2118/165987-MS

[41] M. Icardi, G. Boccardo, D. L. Marchisio, T. Tosco, R. Sethi, Pore-scale simulation of fluid flow and solute dispersion in three-dimensional porous media, Physical Review E 90 (1) (2014) 013032 doi:10.1103/PhysRevE.90.013032. URL http://link.aps.org/doi/10.1103/PhysRevE.90.013032

[42] C. Manwart, U. Aaltosalmi, A. Koponen, R. Hilfer, J. Timonen, Lattice-boltzmann and finitedifference simulations for the permeability for three-dimensional porous media, Physical Review E 
66 (1) (2002) 016702.

[43] M. Osorno, D. Uribe, O. E. Ruiz, H. Steeb, Finite difference calculations of permeability in large domains in a wide porosity range, Archive of Applied Mechanics 85 (8) (2015) 1043-1054.

[44] D. Silin, T. Patzek, A pore-scale model of two-phase flow in water-wet rock, Lawrence Berkeley National Laboratory.

[45] L. Schwartz, N. Martys, D. Bentz, E. Garboczi, S. Torquato, Cross-property relations and permeability estimation in model porous media, Physical Review E 48 (6) (1993) 4584.

[46] L. Cheng, S. Armfield, A simplified marker and cell method for unsteady flows on non-staggered grids, International journal for numerical methods in fluids 21 (1) (1995) 15-34.

[47] I. Barton, R. Kirby, Finite difference scheme for the solution of fluid flow problems on non-staggered grids, International journal for numerical methods in fluids 33 (7) (2000) 939-959.

[48] D. Rempfer, On boundary conditions for incompressible navier-stokes problems, Applied Mechanics Reviews 59 (3) (2006) 107-125.

[49] R. L. Sani, J. Shen, O. Pironneau, P. Gresho, Pressure boundary condition for the time-dependent incompressible navier-stokes equations, International Journal for Numerical Methods in Fluids 50 (6) (2006) 673-682.

[50] W. L. Barth, G. F. Carey, On a boundary condition for pressure-driven laminar flow of incompressible fluids, International journal for numerical methods in fluids 54 (11) (2007) 1313-1325.

[51] M. Ol'Shanskii, V. Staroverov, On simulation of outflow boundary conditions in finite difference calculations for incompressible fluid, International Journal for Numerical Methods in Fluids 33 (4) (2000) 499-534.

[52] J. Nordström, K. Mattsson, C. Swanson, Boundary conditions for a divergence free velocity-pressure formulation of the navier-stokes equations, Journal of Computational Physics 225 (2007) 874-890.

[53] N. Geidarov, Y. N. Zakharov, Y. I. Shokin, Solution of the problem of a viscous fluid flow with a given pressure differential, Russian Journal of Numerical Analysis and Mathematical Modelling 26 (1) (2011) 39-48.

[54] Y. Morinishi, T. Lund, O. Vasilyev, P. Moin, Fully conservative higher order finite difference schemes for incompressible flow, Journal of computational physics 143 (1) (1998) 90-124.

[55] A. J. Chorin, A numerical method for solving incompressible viscous flow problems, Journal of computational physics 2 (1) (1967) 12-26.

[56] R. Mittal, G. Iaccarino, Immersed boundary methods, Annu. Rev. Fluid Mech. 37 (2005) 239-261.

[57] E. Fadlun, R. Verzicco, P. Orlandi, J. Mohd-Yusof, Combined immersed-boundary finite-difference methods for three-dimensional complex flow simulations, Journal of Computational Physics 161 (1) (2000) 35-60

[58] M.-C. Lai, C. S. Peskin, An immersed boundary method with formal second-order accuracy and reduced numerical viscosity, Journal of Computational Physics 160 (2) (2000) 705-719.

[59] I. Malico, P. J. F. de Sousa, Modeling the pore level fluid flow in porous media using the immersed boundary method, in: Numerical Analysis of Heat and Mass Transfer in Porous Media, Springer, 2012, pp. 229-251.

[60] J. D. Hyman, P. K. Smolarkiewicz, C. L. Winter, Heterogeneities of flow in stochastically generated porous media, Physical Review E 86 (5) (2012) 056701.

[61] D. Silin, T. Patzek, Pore space morphology analysis using maximal inscribed spheres, Physica A: Statistical Mechanics and its Applications 371 (2) (2006) 336-360.

[62] M. Hilpert, C. T. Miller, Pore-morphology-based simulation of drainage in totally wetting porous media, Advances in Water Resources 24 (3) (2001) 243-255.

[63] M. A. Knackstedt, R. Sok, S. Adrian, 3D pore scale characterisation of carbonate core: relating pore types and interconnectivity to petrophysical and multiphase flow properties., in: International Petroleum Technology Conference, International Petroleum Technology Conference, 2007.

[64] A. Ghous, F. Bauget, C. Arns, A. Sakellariou, T. Senden, A. Sheppard, R. Sok, W. Pinczewski, R. Harris, G. Beck, M. Knackstedt, Resistivity and permeability anisotropy measured in laminated sands via digital core analysis, in: paper VVV, 46th SPWLA annual logging symposium, 2005.

[65] P. Tomin, I. Lunati, Hybrid Multiscale Finite Volume method for two-phase flow in porous media, Journal of Computational Physics 250 (2013) 293-307. doi:10.1016/j.jcp.2013.05.019. URL http://www.sciencedirect.com/science/article/pii/S0021999113003513

[66] M. L. Porter, M. G. Schaap, D. Wildenschild, Lattice-Boltzmann simulations of the capillary pressure-saturation-interfacial area relationship for porous media, Advances in Water Resources 32 (11) (2009) 1632-1640.

[67] M. Prodanović, S. L. Bryant, A level set method for determining critical curvatures for drainage and imbibition, Journal of colloid and interface science 304 (2) (2006) 442-458. 
[68] M. Prodanovic, S. L. Bryant, Physics-driven interface modeling for drainage and imbibition in fractures, SPE Journal 14 (03) (2009) 532-542.

[69] V. Shabro, M. Prodanovic, C. H. Arns, S. L. Bryant, C. Torres-Verdin, M. A. Knackstedt, Porescale modeling of two-phase flow, in: XVIII International Conference on Computational Methods in Water Resources, Barcelona, 2010.

[70] E. Rodriguez, M. Prodanović, S. L. Bryant, Contact line extraction and length measurements in model sediments and sedimentary rocks, Journal of Colloid and Interface Science 368 (1) (2012) $558-577$.

[71] R. B. Bird, W. E. Stewart, E. N. Lightfoot, Transport Phenomena, 2nd Edition, Wiley New York, 1960.

[72] S. Whitaker, Flow in porous media I: A theoretical derivation of Darcy's law, Transport in porous media 1 (1) (1986) 3-25.

[73] J. C. Strikwerda, Finite difference schemes and partial differential equations, Siam, 2004.

[74] V. Joekar-Niasar, S. Hassanizadeh, A. Leijnse, Insights into the relationships among capillary pressure, saturation, interfacial area and relative permeability using pore-network modeling, Transport in Porous Media 74 (2) (2008) 201-219.

[75] J. Tu, G. H. Yeoh, C. Liu, Computational fluid dynamics: a practical approach, ButterworthHeinemann, 2007.

[76] Y. Saad, Iterative methods for sparse linear systems, Siam, 2003.

[77] H. A. Van der Vorst, Bi-cgstab: A fast and smoothly converging variant of bi-cg for the solution of nonsymmetric linear systems, SIAM Journal on scientific and Statistical Computing 13 (2) (1992) 631-644.

[78] R. C. Aster, B. Borchers, C. H. Thurber, Parameter estimation and inverse problems, Academic Press, 2013.

[79] C. Geuzaine, J.-F. Remacle, Gmsh: A 3-d finite element mesh generator with built-in pre-and postprocessing facilities, International Journal for Numerical Methods in Engineering 79 (11) (2009) $1309-1331$.

[80] U. Ayachit, et al., The paraview guide: A parallel visualization application (2012).

[81] H. G. Weller, G. Tabor, H. Jasak, C. Fureby, A tensorial approach to computational continuum mechanics using object-oriented techniques, Computers in Physics 12 (6) (1998) 620-631. doi: 10.1063/1.168744. URL http://link.aip.org/link/?CIP/12/620/1

[82] M. Kumar, Multiphase flow in reservoir cores using digital core analysis, Australian National University, 2009

[83] R. Nock, F. Nielsen, Statistical region merging, Pattern Analysis and Machine Intelligence, IEEE Transactions on 26 (11) (2004) 1452-1458.

[84] Prodanović, M., http://users.ices.utexas.edu/ masha/lsmpqs/index.html, accessed: Mar 16, 2015.

[85] M. Prodanović, S. L. Bryant, J. S. Davis, Numerical simulation of diagenetic alteration and its effect on residual gas in tight gas sandstones, Transport in Porous Media 96 (1) (2013) 39-62.

[86] http://openmp.org/wp/, accessed: Mar 29, 2015.

[87] http://www.tacc.utexas.edu, accessed: Mar 30, 2015

[88] G. Mavko, A. Nur, The effect of a percolation threshold in the kozeny-carman relation, Geophysics 62 (5) (1997) 1480-1482

[89] A. Tokan-Lawal, M. Prodanović, P. Eichhubl, Investigating flow properties of partially cemented fractures in travis peak formation using image-based pore-scale modeling, Journal of Geophysical Research: Solid Earth.

[90] http://fiji.sc/Fiji, accessed: Mar 30, 2015.

[91] http://www.paraview.org, accessed: Mar 29, 2015. 